\title{
Learning and Stress Shape the Reward Response Patterns of Serotonin Neurons
}

\author{
Weixin Zhong, ${ }^{1,2 \star}$ Yi Li, ${ }^{2 \star}$ Qiru Feng, ${ }^{2,3}$ and $₫$ Minmin Luo ${ }^{1,2,3}$ \\ ${ }^{1}$ Graduate School of Peking Union Medical College, Chinese Academy of Medical Sciences, Beijing 100730, China, ${ }^{2}$ National Institute of Biological Sciences, \\ Beijing 102206, China, and ${ }^{3}$ School of Life Sciences, Tsinghua University, Beijing 100084, China
}

The ability to predict reward promotes animal survival. Both dopamine neurons in the ventral tegmental area and serotonin neurons in the dorsal raphe nucleus (DRN) participate in reward processing. Although the learning effects on dopamine neurons have been extensively characterized, it remains largely unknown how the response of serotonin neurons evolves during learning. Moreover, although stress is known to strongly influence reward-related behavior, we know very little about how stress modulates neuronal reward responses. By monitoring $\mathrm{Ca}^{2+}$ signals during the entire process of Pavlovian conditioning, we here show that learning differentially shapes the response patterns of serotonin neurons and dopamine neurons in mice of either sex. Serotonin neurons gradually develop a slow ramp-up response to the reward-predicting cue, and ultimately remain responsive to the reward, whereas dopamine neurons increase their response to the cue but reduce their response to the reward. For both neuron types, the responses to the cue and the reward depend on reward value, are reversible when the reward is omitted, and are rapidly reinstated by restoring the reward. We also found that stressors including head restraint and fearful context substantially reduce the response strength of both neuron types, to both the cue and the reward. These results reveal the dynamic nature of the reward responses, support the hypothesis that DRN serotonin neurons signal the current likelihood of receiving a net benefit, and suggest that the inhibitory effect of stress on the reward responses of serotonin neurons and dopamine neurons may contribute to stress-induced anhedonia.

Key words: fiber photometry; learning; Pavlovian conditioning; stress-induced anhedonia; stressors; sucrose

\section{Significance Statement}

Both serotonin neurons in the dorsal raphe and dopamine neurons in the ventral tegmental area are intimately involved in reward processing. Using long-term fiber photometry of $\mathrm{Ca}^{2+}$ signals from freely behaving mice, we here show that learning produces a ramp-up activation pattern in serotonin neurons that differs from that in dopamine neurons, indicating complementary roles for these two neuron types in reward processing. Moreover, stress treatment substantially reduces the reward responses of both serotonin neurons and dopamine neurons, suggesting a possible physiological basis for stress-induced anhedonia.

\section{Introduction}

The ability to predict a reward and to adjust reward responses according to behavioral contexts can enable animals to gain rewards at relatively low costs. Reward processing involves dopamine neurons in the ventral tegmental area (VTA) and serotonin

\footnotetext{
Received May 1, 2017; revised July 6, 2017; accepted July 29, 2017.

Author contributions: W.Z., Y.L., and M.L. designed research; W.Z. and Y.L. performed research; W.Z., Y.L., and Q.F. contributed unpublished reagents/analytic tools; W.Z., Y.L., and M.L. analyzed data; W.Z., Y.L., and M.L. wrote the paper.

This work was supported by China MOST (2012YQ03026005, 2013ZX0950910, 2015BAI08B02), NNSFC (91432114, 91632302), and the Beijing Municipal Government to M.L.

*W.Z. and Y.L. contributed equally to this work.

The authors declare no competing financial interests.

Correspondence should be addressed to Dr. Minmin Luo, National Institute of Biological Sciences, \#7 Science Park Road, Zhongguancun Life Science Park, Beijing 102206, China. E-mail: luominmin@nibs.ac.cn.

DOI:10.1523/JNEUROSCI.1181-17.2017

Copyright $@ 2017$ the authors $\quad 0270-6474 / 17 / 378863-13 \$ 15.00 / 0$
}

neurons in the dorsal raphe nucleus (DRN; Schultz et al., 1997; Montague et al., 2004; Nakamura, 2013; Hu, 2016; Luo et al., 2016). VTA dopamine neurons encode reward prediction error (Schultz et al., 1997; Montague et al., 2004; Cohen et al., 2012). DRN serotonin [5-hydroxytryptamine (5-HT)] neurons are activated by various primary rewards before learning and by rewardpredicting cues after learning (Nakamura et al., 2008; BrombergMartin et al., 2010; Miyazaki et al., 2011; Liu et al., 2014; Li et al., 2016; Matias et al., 2017). Unlike dopamine neurons, there is at present no unified theory describing how serotonin neurons contribute to regulating reward-related behaviors (Hayashi et al., 2015; Luo et al., 2016). Previous studies have predominantly focused on serotonin neuronal activity before and after training (Nakamura et al., 2008; Bromberg-Martin et al., 2010; Miyazaki et al., 2011; Li et al., 2016; Matias et al., 2017). It remains unclear how the responses of serotonin neurons evolve during learning. It is also unknown how important reward parameters such as 
reward values and reward availability may differentially affect serotonin neurons and dopamine neurons. Addressing these questions may help elucidate the potentially different roles of serotonin neurons and dopamine neurons in reward-related behaviors.

Very little is known about how stress can influence the reward responses of serotonin neurons and dopamine neurons. Numerous studies have shown at the behavioral level that acute stressors may change reward sensitivity for humans and animals (Bogdan and Pizzagalli, 2006; Born et al., 2010; Porcelli et al., 2012; Berghorst et al., 2013; Kumar et al., 2014). Stress is often associated with anhedonia (Kendler et al., 1999; Gold and Chrousos, 2002; Hammen, 2005). Moreover, limited ability to adapt to stressful contexts causes defects in emotion control, and may lead to psychological disorders such as depression, anxiety, and post-traumatic stress disorders (Schneiderman et al., 2005; de Kloet et al., 2005; Chrousos, 2009). Although it is important for animals to properly adjust reward responses according to stress levels, how stress modulates reward-associated neuronal activity remains unclear. Stress coping involves dopamine neurons and serotonin neurons (Anisman and Zacharko, 1990; Amat et al., 2005; Bekris et al., 2005; Porcelli et al., 2012; Hollon et al., 2015). Both VTA dopamine neurons and DRN serotonin neurons receive strong inputs from the lateral habenula that respond to stressors (WatabeUchida et al., 2012; Pollak Dorocic et al., 2014; Weissbourd et al., 2014; Wang et al., 2017). However, it remains unclear how acute stress modulates the responses of DRN serotonin neurons and VTA dopamine neurons to rewards and to reward-predicting cues.

Here, we used fiber photometry of $\mathrm{Ca}^{2+}$ signals to examine how learning and acute stressors modulate the reward responses of DRN serotonin neurons and VTA dopamine neurons (Chen et al., 2013; Gunaydin et al., 2014; Li et al., 2016). We first tracked the activity patterns of serotonin neurons in freely behaving mice throughout the course of appetitive Pavlovian conditioning. We then compared the reward response pattern of DRN serotonin neurons to that of VTA dopamine neurons. We further examined the effects of reward values, omission, and reinstatement on both neuron populations. We finally studied the effects of acute stressors on the reward responses of DRN serotonin neurons and VTA dopamine neurons. These experiments revealed that learning differentially shapes the response patterns of both neuron types. Moreover, acute stress reduces the extent of activation associated with both rewards and reward-predicting cues. Our results indicate that serotonin neurons and dopamine neurons play complementary roles in reward processing. Moreover, acute stress may negatively affect reward processing by suppressing the responses of both serotonin neurons and dopamine neurons.

\section{Materials and Methods}

\section{Subjects}

Animal care and use conformed to the institutional guidelines of the National Institute of Biological Sciences, Beijing, as well as the governmental regulations of China. We used adult (8-14 weeks old at the time of surgery) SERT-Cre mice [strain name B6.Cg-Tg(Slc6a4-Cre)ET33Gsat; MMRRC; RRID: MGI_3691580] and DAT-Cre mice [strain name B6.SJLSlc6a3tm1.1(cre)Bkmn; The Jackson Laboratory; RRID: MGI_3689567] of either sex. Mice were maintained with a 12/12 photoperiod (lights on at 8:00 A.M.) and were housed in groups of five for $6-8$ weeks. After surgery, mice were housed individually with a reversed photoperiod (lights off at 8:00 A.M.) for at least $10 \mathrm{~d}$ before conducting any further experiments.

\section{Surgery and virus injection}

Details for the construction of pAAV-DIO-GCaMP6m plasmid and packing of the AAV vectors have been described previously (Li et al., 2016). To inject the AAV vectors to specific brain regions, mice were anesthetized with pentobarbital (i.p., $80 \mathrm{mg} / \mathrm{kg}$ ) and mounted to a stereotaxic apparatus. After making a small craniotomy, we slowly injected viral vectors ( $500 \mathrm{~nL}$ at $40 \mathrm{~nL} / \mathrm{min}$ ) into the DRN (coordinates: - 5.2/0/ $2.5 \mathrm{~mm}$; AP/ML/DV) with a $15^{\circ}$ caudal-to-rostral angle or the VTA (coordinates: $-3.2 / 0.55 / 4.6 \mathrm{~mm}$; AP/ML/DV) using a glass pipette controlled by a microsyringe pump (Nanoliter 2000 Injector, WPI). The glass pipette was left in place for 10 additional minutes and then slowly withdrawn. Following AAV-DIO-GCaMP6m virus injection, an optical fiber [230 $\mu$ m outer diameter (o.d.), $0.37 \mathrm{NA}$; Shanghai Fiblaser] was placed in a ceramic ferrule and was inserted toward the DRN or VTA. The ceramic ferrule was supported with a skull-penetrating M1 screw and dental acrylic.

We implanted intraoral cheek fistula using a previously described procedure (Hintiryan et al., 2006). Four days after AAV injection, we made an incision in the mouse cheek that was lateral and rostral to the first molar and made another incision in the scalp. We inserted soft a Silastic tube [ $30 \mathrm{~mm}$ in length, $0.30 \mathrm{~mm}$ inner diameter (i.d.) and $0.46 \mathrm{~mm}$ o.d.; Dow Corning] $2 \mathrm{~mm}$ into the oral cavity through the cheek incision site, and then guided the tube out subcutaneously through the scalp incision. An L-shaped 26 gauge (o.d. $0.48 \mathrm{~mm}$ ) stainless steel tube was connected to the Silastic tube and was imbedded alongside the ceramic ferrule containing the optical fiber. A piece of polyethylene tubing $(10 \mathrm{~mm}$ in length, $0.4 \mathrm{~mm}$ i.d., $1.1 \mathrm{~mm}$ o.d.) was fit to the exposed end of the L-shaped steel tube. When the tube system was not being used in an experiment, a plug was inserted to the exposed end of the polyethylene tube to prevent blockage. After the implantation of intraoral cheek fistula, mice were housed individually.

\section{Fiber photometry}

The fiber photometry experimental device/setup used here was described in detail previously (Gunaydin et al., 2014; Guo et al., 2015). Briefly, an exciting laser beam from a $488 \mathrm{~nm}$ laser (OBIS 488LS, Coherent) was first reflected by a dichroic mirror (MD498, Thorlabs), focused with a 10X objective lens ( $\mathrm{NA}=0.3$; Olympus), and coupled to an optical fiber patch cable $(230 \mu$ m o.d., $\mathrm{NA}=0.37 ; 2 \mathrm{~m}$ long). A patch cable was then connected to the optical cannula implanted in the brain through a mating sleeve. The laser power at the tip of optical fiber was adjusted to $0.03-0.04 \mathrm{~mW}$ to minimize bleaching of GCaMP probes. The excited GCaMP fluorescence was transmitted back through the optical fiber and objective lens. After passing the dichroic mirror and a bandpass filter (MF525-39, Thorlabs), the fluorescence signal was converted to an electrical signal by a photomultiplier tube (R3896, Hamamatsu). The electrical signal was then amplified and further low-pass filtered ( $40 \mathrm{~Hz}$ cutoff; Brownlee 440). The analog voltage signals were digitalized at $500 \mathrm{~Hz}$ using a Power 1401 digitizer and recorded with Spike2 software (CED).

\section{Behavioral tasks}

Appetitive classical conditioning. For Pavlovian conditioning, mice were deprived of water for $24 \mathrm{~h}$ before training. In each of the conditioning session, a mouse was repetitively presented with 100 trials of cue-sucrose "pairs" as follows: a 2 s tone [conditioned stimulus (CS)] followed by a $1 \mathrm{~s}$ delay and then the delivery of a $0.5 \mathrm{~s}$ sucrose solution [unconditioned stimulus (US), detailed below], with 20-40 s randomized intertrial intervals. Auditory tones were generated with square waveforms ( 0.5 duty, $4 \mathrm{kHz}$; digitized at $96 \mathrm{kHz}$ ) and delivered via speakers. A peristaltic pump infused the sucrose solution $(5 \% \mathrm{w} / \mathrm{v})$ into the oral cavity through the intraoral cheek fistula at the speed of $20 \mu \mathrm{l} \cdot \mathrm{s}^{-1}$. The entire conditioning process consisted of at least four and no more than six daily sessions for the SERT-Cre mice and six daily sessions for the DAT-Cre mice. A MATLAB program and an Arduino R3 controller were used to generate the tone signal and the pump trigger signal; a Power 1401 digitizer simultaneously recorded tone timing (100 $\mathrm{Hz}$ sampling frequency) and GCaMP signals $(500 \mathrm{~Hz})$. At the end of the session, the animal was returned to its home cage and was allowed to access water freely for 5 $\min$. On the day after the final conditioning session, we challenged mice with an omission session in which sucrose was omitted during trials 51-150 of a total of 250 trials. 
For training sessions with two reward sizes, mice were exposed to two types of auditory tones, a $2 \mathrm{~s} 4 \mathrm{kHz}$ period of pure tone or a $2 \mathrm{~s}$ period of white noise, that were coupled, respectively, with small and large rewards. The reward sizes were controlled by using two different pump durations $(0.5 \mathrm{vs} 2.5 \mathrm{~s})$ with the same speed $\left(10 \mu \mathrm{l} \cdot \mathrm{s}^{-1}\right)$. For both reward sizes, the sucrose was delivered $1 \mathrm{~s}$ after the end of either auditory tone. The entire training process consisted of a total of six sessions, each of which consisted of 50 pure tone/small reward pairs and 50 white noise/ large reward pairs that were randomly delivered.

Stress. We used quinine, acute head restraint, and footshock as stressors. To exclude the potential effect(s) of water and energy in the sucrose solution, mice were allowed ad libitum access water and food before tests. For all of the stressor treatments, mice were given 20 normal trials ("A"), 20 stress trials ("B"), and then 20 additional normal trials ("A") in an $A B A$ design. We first placed a mouse in a chamber and recorded $\mathrm{Ca}^{2+}$ signals during an initial round of 20 trials of random intraoral infusion of a sucrose solution. To examine the effect of quinine addition (stress), we replaced the sucrose solution (5\% sucrose) with the mixture of sucrose ( $5 \%$ sucrose) and quinine $(10 \mathrm{~mm})$ for the next 20 trials. The final 20 trials used unaltered sucrose solution. To test the effect of acute head restraint, we restrained the mouse by fixing it to a standing platform with a small horizontal bar implanted on its head. The initial 20 trials of these experiments examined the responses of freely moving mice to the random intraoral infusion of sucrose. For the 20 stressed trials, mice were restrained on the standing platform during the sucrose infusion trials. The mouse was then released and returned to the neutral chamber for the final 20 unstressed sucrose infusion trials. To examine the effect of a fearful context on sucrose-evoked $\mathrm{Ca}^{2+}$ responses, we induced stress using footshocks. After 20 trials of recordings in a neutral chamber, the mouse was introduced to an opaque acrylic box with a metal grid floor (footshock context, $25 \mathrm{~L} \times 25 \mathrm{~W} \times 30 \mathrm{H} \mathrm{in} \mathrm{cm}$ ). After giving five random footshocks $(0.7 \mathrm{~mA}, 0.5-1 \mathrm{~s})$ within a $10 \mathrm{~min}$ period, we waited $\sim 10 \mathrm{~min}$ and then recorded $\mathrm{Ca}^{2+}$ responses to sucrose for 20 trials. We finally placed the mouse back to the initial chamber for 20 unstressed trials. Similar procedures were performed to examine the effects of stress on cue-reward conditioned responses, with the exception that a mouse was fully conditioned via either 400 or 600 trials of cue-reward coupling. During these cue-reward conditioned response experiments, the tone/ reward pairs were presented during the stress sessions.

Aversive classical conditioning. The entire conditioning session consisted of 100 trials that paired a tone with quinine. We used a $12 \mathrm{kHz}$ pure tone as the conditioned stimulus and quinine solution $(10 \mathrm{~mm}, 10 \mu \mathrm{l}$ in $0.5 \mathrm{~s}$ ) as the unconditioned stimulus.

\section{Immunostaining}

Mice were deeply anesthetized with an overdose of pentobarbital and then intracardially perfused with $0.9 \%$ saline followed by $4 \%$ paraformaldehyde in PBS. After cryoprotection with 30\% sucrose, mouse brains were sectioned coronally ( $35 \mu \mathrm{m}$ thick) with a cryostat (Leica CM1900). For immunohistochemistry, the sections were blocked with 3\% BSA in PBS with $0.3 \%$ Triton $\mathrm{X}-100$. We incubated brain sections with a rabbit polyclonal antibody against tryptophan hydroxylase 2 (TPH2; 1:400; Millipore, ABN60) or a rabbit polyclonal antibody against TH (1:500; Millipore, $\mathrm{AB} 152)$ at $4^{\circ} \mathrm{C}$ overnight. After washing with $\mathrm{PBS}$, the brain sections were incubated with Cy3-conjugated goat anti-rabbit IgG (1:500; Jackson ImmunoResearch) for $1 \mathrm{~h}$ at room temperature. PBS-washed sections were then coverslipped with $50 \%$ glycerol mounting medium. The stained sections were imaged with a confocal microscope (LSM510 META or Axiovert 200M, Zeiss).

\section{Data analysis and statistical tests}

No data were excluded. Photometry data were exported from the Spike2 program as MATLAB .mat files for further analysis. After smoothing the data with a moving average filter (20 ms span), we segmented the data based on behavioral events within individual trials. We derived the fluorescence change values $(\Delta F / F)$ by calculating $\left(F-F_{0}\right) / F_{0}$, where $F_{0}$ is the baseline fluorescence signal averaged over a $1.5 \mathrm{~s}$ long control time window; this window was typically commenced $0.5 \mathrm{~s}$ before initiating a trigger event (cue or infusion). $\Delta F / F$ values are presented as heatmaps or as peri-event plots.
To examine changes in response strengths during the anticipatory phase and the consummatory phase of the reward conditioning, we normalized the response strengths of each individual trial across the appetitive conditioning sessions. For DRN serotonin neurons, we initially calculated the average of the peri-event $\mathrm{Ca}^{2+}$ signals of the first 50 trials in the initial session; we identified the peak value in the average plot, and normalized the response strength of each of the trials to the peak value. For normalization of VTA dopamine neuron data, we used the sum of $\mathrm{Ca}^{2+}$ signals during reward expectation $(0-3 \mathrm{~s})$ and reward acquisition (3-5s) in the peri-event plot. We then averaged the normalized response strength during the anticipatory phase and consummatory phase for every 10 trials. To measure the response strength of both serotonin and dopamine neurons during the consummatory phase, we determined the peak value of the normalized peri-event data during the $2 \mathrm{~s}$ time window following reward delivery ( $3-5 \mathrm{~s}$ following the cue onset). To examine the response strength of serotonin neurons during the reward anticipatory phase, we calculated the area under the peri-event plot during the $3 \mathrm{~s} \mathrm{time}$ window following cue onset ( $0-3 \mathrm{~s}$ following the cue onset) and averaged the value per 10 trials. For dopamine neurons, we normalized the peak response intensity during the anticipatory phase to the sum of the peaks during the anticipatory phase and the consummatory phase. To analyze the discriminability of reward values and their associated cues, we used the receiver operating characteristic (ROC) and calculated the area under the ROC curve (auROC). An auROC value of 0.5 indicates that there was no difference in activity in response to large reward versus small reward.

We used permutation tests to analyze the statistical significance of the event-related fluorescence change (Groppe et al., 2011). We used 5000 permutations for an $\alpha$ level of 0.001 to compare the values of $\Delta F / F$ at each time point with the event-related fluorescence (ERF) baseline values. The $99.9 \%$ fraction of the baseline $\Delta F / F$ values was defined as the upper threshold value, and the $0.1 \%$ fraction was defined as the lower threshold. Values above the upper threshold or below the lower threshold were considered significant increase or decrease, respectively. Statistical results were superimposed on the average ERF curve with red and blue lines indicating statistically significant $(p<0.001)$ increases or decreases, respectively. The duration of activation and inhibition was respectively calculated by summing the total time during which the $\mathrm{Ca}^{2+}$ signals were above the upper-threshold or below the lower threshold.

\section{Results \\ Pavlovian conditioning differentially shapes the response patterns of DRN serotonin neurons and VTA dopamine neurons}

We first investigated how the activity of DRN serotonin neurons changes during learning. We trained mice with Pavlovian conditioning by repetitively presenting an auditory cue (CS) with delayed delivery of a sucrose solution (US; Fig. 1A). Within each trial (100 trials per session), a $2 \mathrm{~s}$ tone was followed by a $1 \mathrm{~s}$ delay and then direct infusion of sucrose solution (10 $\mu \mathrm{l}$ in $0.5 \mathrm{~s})$ into the oral cavity through a small implanted tube (Fig. $1 B$ ). To monitor $\mathrm{Ca}^{2+}$ signals in DRN serotonin neurons, we expressed a genetically encoded $\mathrm{Ca}^{2+}$ indicator, GCaMP6, in serotonin neurons by infusing the Cre-dependent adeno-associated virus AAVDIO-GCaMP6m into the DRN of SERT-Cre mice (henceforth referred to as SERT-DRN-GCaMP6 mice). This resulted in the highly accurate and efficient expression of GCaMP6 in neurons expressing $\mathrm{TPH} 2$, the rate-limiting enzyme for serotonin synthesis in the brain (Zhang et al., 2004; Li et al., 2016; Fig. 1C). We implanted an optical fiber with its tip in the DRN for fiber photometry of GCaMP fluorescence changes in serotonin neuron populations during the entire conditioning process (Fig. $1 D$ and Fig. 1-1 $A, B$ available at https://doi.org/10.1523/JNEUROSCI. 1181-17.2017.f1-1). Our previous study demonstrated that the GCaMP fluorescence changes reflect $\mathrm{Ca}^{2+}$ signals rather than any artifact of movement (Li et al., 2016). 
A

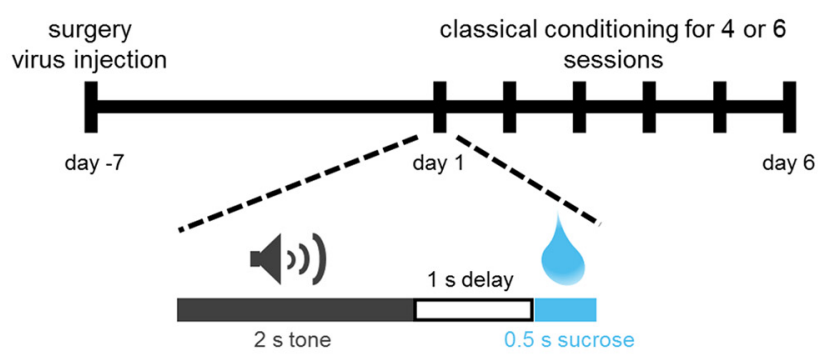

C
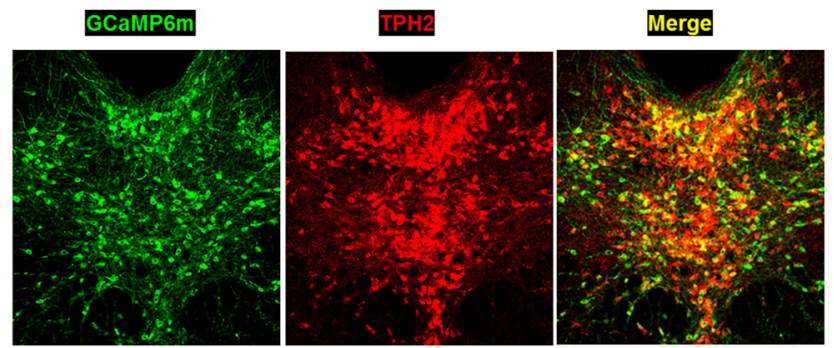

E

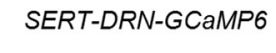

())
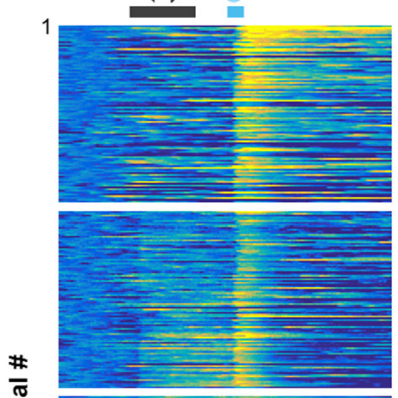

$\stackrel{\frac{\pi}{2}}{\frac{\pi}{2}}$

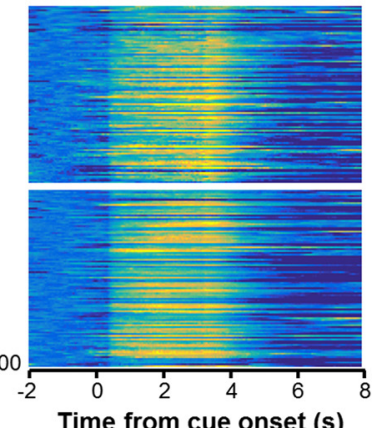

Time from cue onset (s)

G

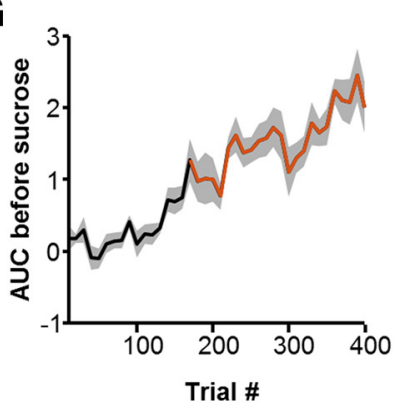

F SERT-DRN-GCaMPG
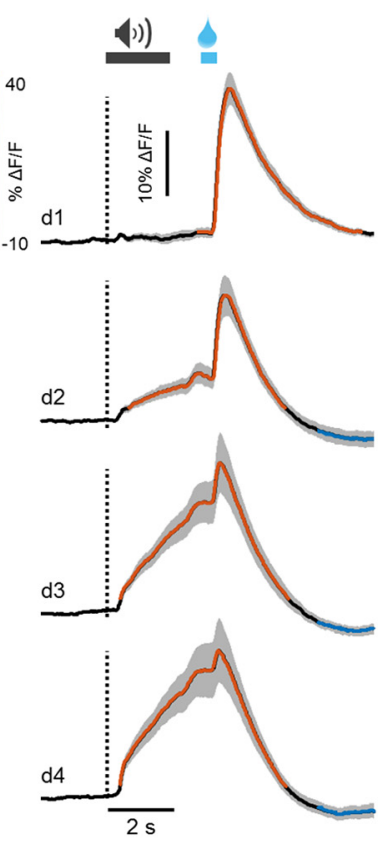

H

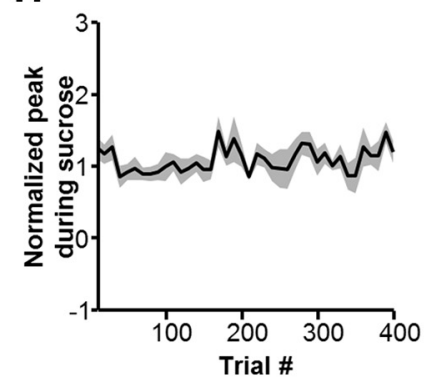

B

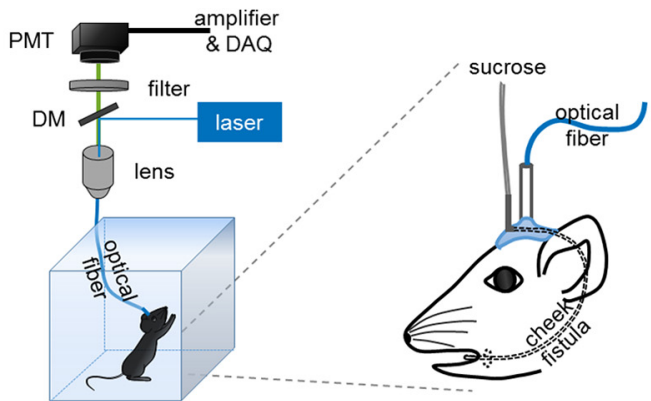

D

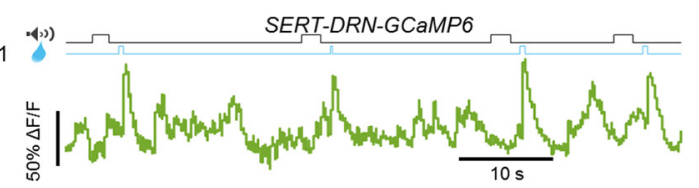

d6

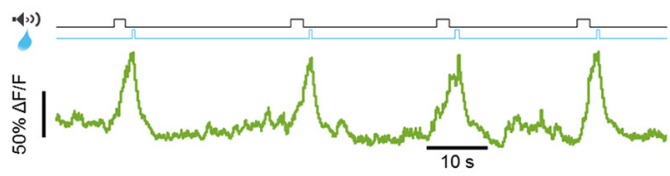

DAT-VTA-GCaMP6

A))
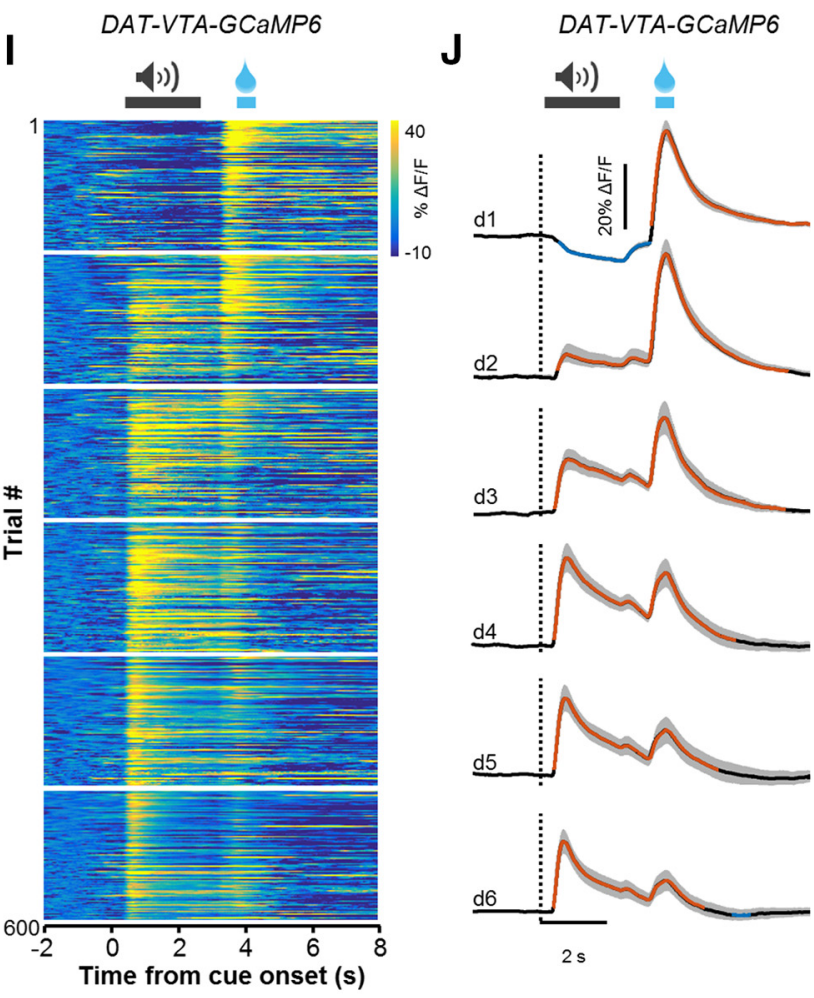

K

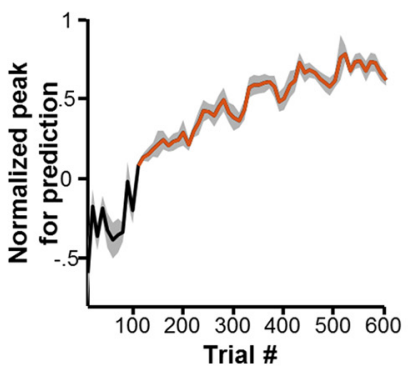

$\mathbf{L}$

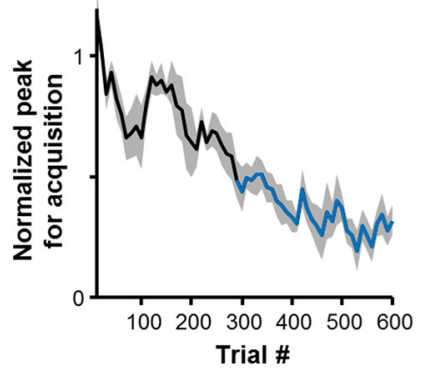

Figure 1. Appetitive Pavlovian conditioning differentially shapes the response patterns of DRN serotonin neurons and VTA dopamine neurons. $A$, The design of the cue-reward classical conditioning task. An auditory tone ( $2 \mathrm{~s}$ ) was repetitively paired with sucrose delivery $(0.5 \mathrm{~s})$ after a $1 \mathrm{~s}$ delay. Each daily session consisted of 100 trials, and each mouse completed four or six sessions. $B$, Fiber photometry of $\mathrm{Ca}^{2+}$ signals from freely behaving mice engaged in the classical conditioning task. We infused sucrose solution into the oral cavity through cheek fistula as indicated in the schematic. We implanted an optical fiber into the DRN to simultaneously record changes in GCaMP6 fluorescence using fiber photometry. C, Expression of (Figure legend continues.) 
We analyzed how the changes in the $\mathrm{Ca}^{2+}$ signal patterns related to the timing of the cue and of sucrose delivery for all trials in all of the sessions. We segmented raw traces and aligned the $\mathrm{Ca}^{2+}$ signals to the cue onset (Fig. $1 E$ ). We then averaged the signals to plot peri-event time histograms for individual mice, and calculated the mean $\mathrm{Ca}^{2+}$ signals for each conditioning session $(n=14$ mice; Fig. $1 F$ and Fig. $1-1 B$ available at https://doi. org/10.1523/JNEUROSCI.1181-17.2017.f1-1). In the second session, as the number of trials increased, the cue began to elicit an increasing trend in the intensity of $\mathrm{Ca}^{2+}$ signals ("ramp-up" trend); these cue-evoked $\mathrm{Ca}^{2+}$ signals lasted through the delay period and peaked immediately following sucrose infusion. The activation of serotonin neurons during the anticipatory phase (including the tone and the delay period) reached statistical significance following $\sim 170$ trials of training $(p<0.05$; permutation test; Fig. $1 G$ ). In the subsequent conditioning sessions, the intensity of these cue-evoked ramp-up $\mathrm{Ca}^{2+}$ signals continued to increase (Fig. $1 F, G$ ). By the end of the conditioning session, the maximal amplitude of the cue-evoked $\mathrm{Ca}^{2+}$ signals was eventually as high as the amplitude of the sucrose-evoked $\mathrm{Ca}^{2+}$ signals (Fig. $1 F$ and Fig. $1-1 C$ available at https://doi.org/10.1523/ JNEUROSCI.1181-17.2017.f1-1). In contrast to the increase of cue-evoked responses, the $\mathrm{Ca}^{2+}$ signals associated with sucrose infusion remained stable throughout the conditioning process (Fig. $1 H$ ). Therefore, learning induces a ramp-up activity pattern in DRN serotonin neurons in response to the CS but does not affect DRN serotonin neuron responses to the US.

It was recently shown that stimulating DRN serotonin neurons slows down animal locomotion (Correia et al., 2017). We analyzed the relationship between the $\mathrm{Ca}^{2+}$ signals and locomotor activity. During the initial phase of the conditioning, mice displayed decreased locomotion upon sucrose delivery. This decrease in locomotion became more pronounced as the conditioning continued; its timing gradually shifted closer to the cue and became stable after three or four conditioning sessions (Fig. 1-1D available at https://doi.org/10.1523/JNEUROSCI.1181-17.2017. f1-1). The onset of $\mathrm{Ca}^{2+}$ signals after the cue preceded the onset of locomotion decrease for $\sim 1 \mathrm{~s}$ (Fig. 1-1D available at https:// doi.org/10.1523/JNEUROSCI.1181-17.2017.f1-1). This suggests that reward anticipation and acquisition, but not a decrease in

$\leftarrow$

(Figure legend continued.) GCaMP6 (Green) in TPH2-immunopositive neurons (red) in the DRN of a SERT-DRN-GCaMP6 mouse. D, Raw traces showing the behavior events of the tone (black), sucrose delivery (blue), and GCaMP6 signals (green) during the conditioning session of day 1 (d1) and day 6 (d6) from a SERT-DRN-GCaMP6 mouse. $E$, Heatmaps illustrating the GCaMP changes $(\triangle F / F, \%)$ of $D R N$ serotonin neurons across six daily classical conditioning sessions from a SERT-DRN-GCaMP6 mouse. $\boldsymbol{F}$, Peri-event plots of the average $\mathrm{Ca}^{2+}$ signals. $\boldsymbol{G}$, The intensity of the $\mathrm{Ca}^{2+}$ signal during the anticipatory phase of DRN serotonin neurons gradually increased as the number of conditioning sessions increased. We measured the area under the peri-event curve (AUC) between cue onset ( $0 \mathrm{~s}$ ) and sucrose delivery ( $3 \mathrm{~s})$ to represent the response strength in the reward anticipatory phase. $\boldsymbol{H}$, The response to sucrose remained relatively unchanged over time. Within each session, the peak response intensity following sucrose delivery $(3-5 \mathrm{~s})$ was normalized to the sucrose response intensities of the initial 50 trials of the conditioning. I, Heatmaps illustrating the GCaMP changes of VTA dopamine neurons during six daily classical conditioning sessions from a DAT-VTA-GCaMP6 mouse. J, Peri-event plots of the average intensity of $\mathrm{Ca}^{2+}$ signals of dopamine neurons. $\boldsymbol{K}, \boldsymbol{L}$, The response strength of VTA dopamine neurons to the tone onset $(\boldsymbol{K})$ and sucrose delivery $(\boldsymbol{L})$ across conditioning sessions (see Figs. 1-1 and 1-2 available at https://doi.org/10.1523/JNEUROSCI.1181-17.2017.f1-1 and https://doi.org/10.1523/JNEUROSCI.1181-17.2017.f1-2, respectively). Shaded areas in $\boldsymbol{F}-\boldsymbol{H}$ and $\boldsymbol{J}-\boldsymbol{L}$ indicate SEM $(\boldsymbol{F}-\boldsymbol{H}, n=14$ SERT-DRN-GCaMP6 mice; $\boldsymbol{J} \boldsymbol{L}, n=9$ DAT-VTA-GCaMP6 mice). $\boldsymbol{F}, \boldsymbol{G}, \boldsymbol{J}-\boldsymbol{L}$, Red and blue colors superimposed on the black line indicate significant increases and decreases from the baseline, respectively $(\boldsymbol{F}, \boldsymbol{J}, \boldsymbol{p}<0.001 ; \boldsymbol{G}, \boldsymbol{H}, \boldsymbol{K}, \boldsymbol{L}, \boldsymbol{p}<0.05$, multivariate permutation tests). locomotor activity, causes the activation of DRN serotonin neurons.

Although the reward response patterns of VTA dopamine neurons have been researched extensively (Schultz et al., 1997; Montague et al., 2004; Matsumoto and Hikosaka, 2009; Cohen et al., 2012; Eshel et al., 2015; Menegas et al., 2017), most studies have taken recordings from head-fixed animals that acquired sucrose by active licking. To enable a direct comparison with the responses of serotonin neurons, we also monitored the $\mathrm{Ca}^{2+}$ response patterns of dopamine neurons in freely behaving mice. Additionally, we delivered sucrose via direct intraoral infusion. After expressing GCaMP6 in VTA dopamine neurons, we recorded the $\mathrm{Ca}^{2+}$ signals of the dopamine neurons from DATVTA-GCaMP mice in six daily sessions, each of which consisted of 100 trials (Fig. 1-2 available at https://doi.org/10.1523/ JNEUROSCI.1181-17.2017.f1-2). In the initial conditioning session, the auditory cue produced a mild yet statistically significant decrease in $\mathrm{Ca}^{2+}$ signals, whereas sucrose infusion strongly activated the dopamine neurons (Fig. $1 I, J$ ). As the conditioning process proceeded, the initially observed cue-evoked inhibition gradually switched, becoming an activation that increased in strength with additional sessions. This cue-evoked activation peaked at $\sim 0.3 \mathrm{~s}$ after cue onset and quickly decayed before the cue ceased (Fig. $1 J$ ). In contrast, the response to sucrose infusion ("reward-associated response") gradually decreased as the conditioning proceeded (Fig. $1 I, J, L$ ). At the population level, the cue-evoked excitatory response was significantly above the baseline after $\sim 110$ trials $(p<0.05$, permutation test; Fig. $1 K$ ), whereas the reward-associated response was significantly below the initial value after $\sim 290$ trials $(p<0.05$, permutation test), and was eventually reduced to $\sim 25 \%$ of its original amplitude (Fig. $1 \mathrm{~J}, L$ ).

It was thus clear at the end of the learning process that VTA dopamine neurons differed from DRN serotonin neurons in two key ways. First, the cue evoked a gradually increasing ramp-up $\mathrm{Ca}^{2+}$ signal that lasted throughout the reward anticipatory phase in serotonin neurons, whereas the cue only evoked a brief excitatory response in dopamine neurons. Second, the reward continued to elicit $\mathrm{Ca}^{2+}$ signals in serotonin neurons throughout the learning period, whereas the reward gradually became ineffective for activating dopamine neurons. We noted that our observations of the response patterns of DRN serotonin neurons differed from a recent study which showed that the $\mathrm{Ca}^{2+}$ signals of serotonin neurons in head-fixed mice transiently increased following the reward-predicting cue and decreased following sucrose licking (Matias et al., 2017). Moreover, this recent study reported that serotonin neurons were activated when a fully predicted reward was omitted.

We investigated how changes in reward availability affected the activation patterns of DRN serotonin neurons in freely behaving mice. Following six sessions of Pavlovian conditioning, the mice were tested with an "omission session" during which we presented cue-reward pairs in the initial 50 trials, omitted sucrose from the pair in the following 100 trials, and reinstated sucrose in the final 100 trials (Fig. $2 A$ ). In $<20$ trials, reward omission completely abolished the excitatory responses of DRN serotonin neurons during both the reward anticipatory phase $(0-3 \mathrm{~s} ;$ Fig. $2 \mathrm{~B})$ and the previous reward consumption phase (3-5 s; Fig. 2C). Following reward reinstatement, the sucrose-evoked responses in DRN serotonin neurons returned immediately (Fig. $2 C)$; the cue-evoked responses recovered fully within 20 trials, which was much more quickly than the number of trials $(\sim 170)$ that were required to establish the cue responses during the initial 
A

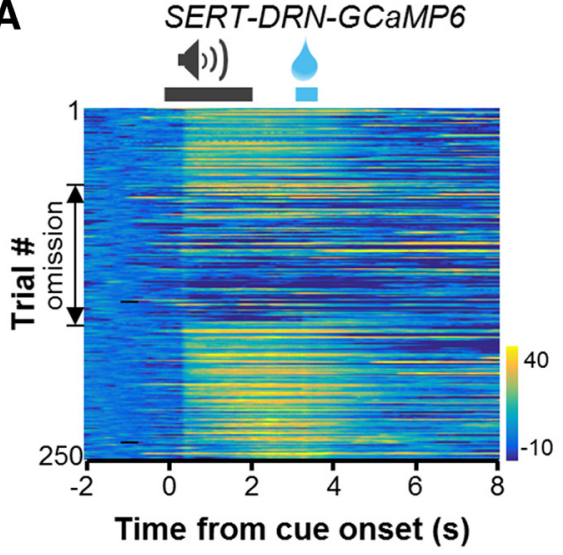

D

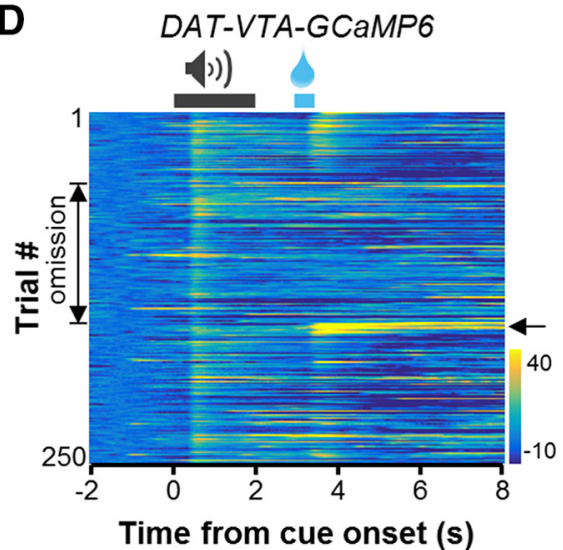

B

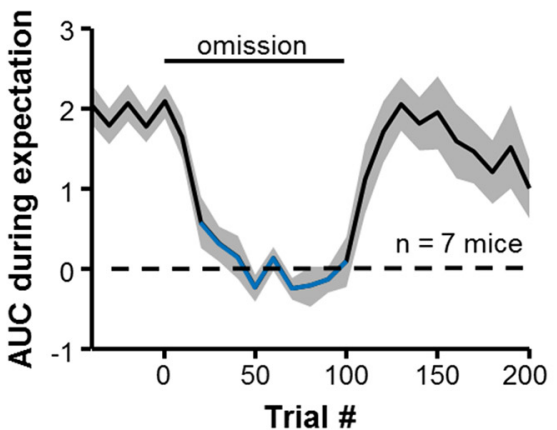

E

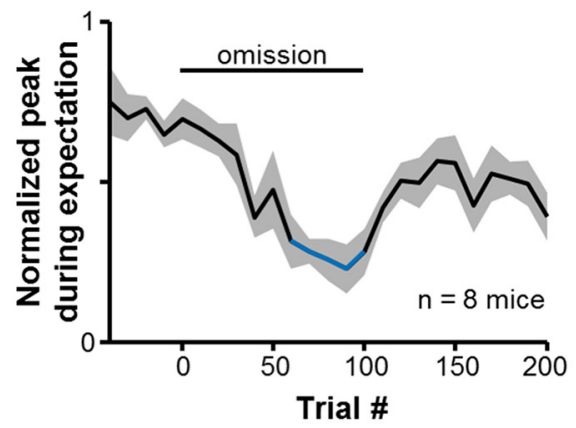

C SERT-DRN-GCaMP6

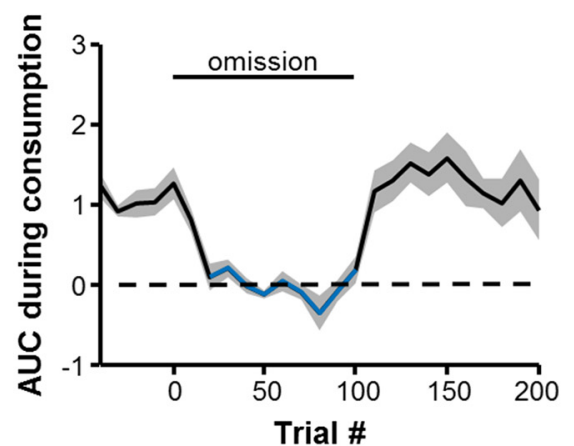

$\mathbf{F}$

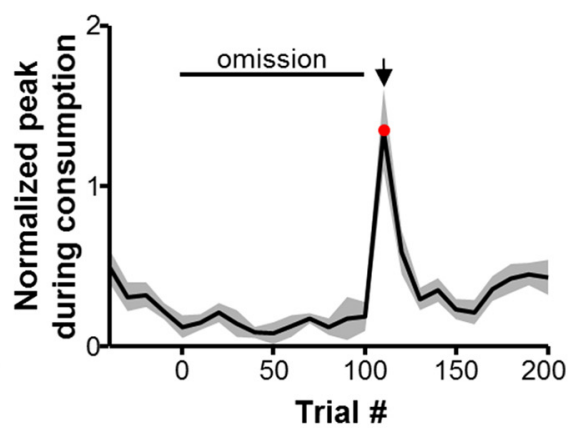

Figure 2. The effects of reward omission and reinstatement on the responses of serotonin neurons and dopamine neurons. $A, A$ heatmap showing the $\mathrm{Ca}^{2+}$ signals of DRN serotonin neurons in a mouse challenged with reward omission. We presented the CS for all of the trials, but omitted US during trials 51-150. $B$, The effect of reward omission on the activity of serotonin neurons during the anticipatory phase ( $n=7$ SERT-DRN-GCaMP6 mice). Each point in the line plot represents the average value of the normalized area under the curve (AUC) between cue onset $(0 s)$ and sucrose delivery $(3 \mathrm{~s})$ for 10 trials. $\boldsymbol{C}$, The effect of reward omission on the activity of serotonin neurons during the reward consumption phase ( $n=7$ SERT-DRN-GCaMP6 mice). D-F, The effect of reward omission on the activity of VTA dopamine neurons ( $n=9$ DAT-VTA-GCaMP6 mice). We defined response strength in $\boldsymbol{E}$ and $\boldsymbol{F}$ as the peak value normalized to the average peak value of the initial 50 trials during the first session. Shaded areas in $\boldsymbol{B}, \boldsymbol{C}, \boldsymbol{E}$, and $\boldsymbol{F}$ indicate $\mathrm{SEM}$. Red and blue colors in $\boldsymbol{B}, \boldsymbol{C}, \boldsymbol{E}$, and $\boldsymbol{F}$ indicate significant increases and decreases from the baseline, respectively $(p<0.05$, multivariate permutation test).

conditioning phase (Fig. 2B). In VTA dopamine neurons, reward omission decreased cue-evoked responses as was seen in DRN serotonin neurons, but it took $\sim 60$ trials for this decrease to reach statistical significance (Fig. 2E). Possibly because the population GCaMP signals were not strong enough to report a mild decrease in spiking frequency, we did not observe the inhibitory responses that have previously been associated with negative prediction error as caused by reward omission (Hollerman and Schultz, 1998). When the sucrose was reintroduced, the responses of VTA dopamine neurons to sucrose increased dramatically for $\sim 10$ trials and then quickly decreased to the pre-omission level (Fig. 2D,F, arrows). The recovery of the cue-evoked response in VTA dopamine neurons following reward reinstatement required $\sim 20$ trials and was thus much slower than the recovery that we had observed in DRN serotonin neurons (Fig. 2E). Therefore, both DRN serotonin neurons and VTA dopamine neurons adapt their responses according to reward availability. Serotonin neurons rapidly and faithfully tracked reward availability, while VTA dopamine neurons respond strongly to unexpected reward availability.

Serotonin neurons and dopamine neurons respond in a reward value-dependent manner

Previously, we showed that quinine itself does not significantly inhibit DRN serotonin neurons (Li et al., 2016). However, it re- mains to be tested whether adding the bitter tastant quinine to the sucrose solutions would affect the responses of DRN serotonin neurons to sucrose. In a single quinine session of 60 trials, we recorded the responses to sucrose (5\%) during the initial 20 trials. We then mixed quinine with sucrose solution while maintaining the sucrose concentration for the next 20 trials. In the final 20 trials, we delivered sucrose solution without quinine. Adding quinine into sucrose solution completely and reversibly abolished sucroseevoked $\mathrm{Ca}^{2+}$ signals $\left(n=6\right.$ mice; Fig. $3 A-C ; F_{(2,15)}=10.33, p=$ 0.0017 , evaluated via repeated-measures one-way ANOVA with "aversive stimuli" as the within-subject factor). Adding quinine into sucrose solution also completely abolished the sucroseassociated $\mathrm{Ca}^{2+}$ signals of VTA dopamine neurons $(n=7$ mice; Fig. $3 D-F ; F_{(2,18)}=12.29, p=0.0003$, repeated-measures oneway ANOVA with aversive stimuli as the within-subject factor). Although quinine itself has a mildly inhibitory effect on the baseline activity of both serotonin neurons and dopamine neurons, coupling a cue to quinine resulted in a statistically significant decrease in the $\mathrm{Ca}^{2+}$ signals during the cue (Figs. 3-1 and 3-2 available at https://doi.org/10.1523/JNEUROSCI.1181-17.2017. f3-1 and https://doi.org/10.1523/JNEUROSCI.1181-17.2017. f3-2, respectively), demonstrating the aversive nature of quinine. Thus, aversive stimuli reduce the reward value for both DRN serotonin neurons and VTA dopamine neurons. 
A

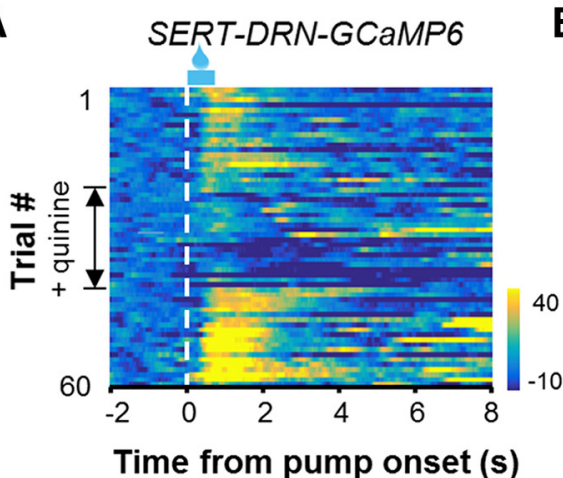

B

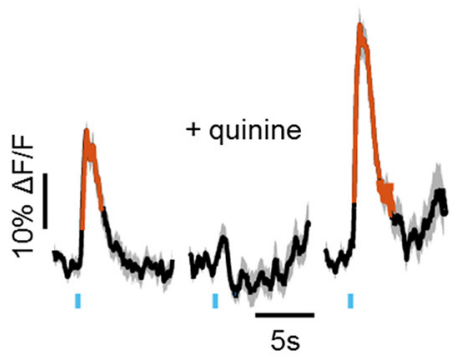

C

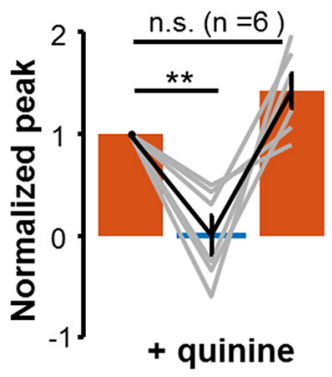

D

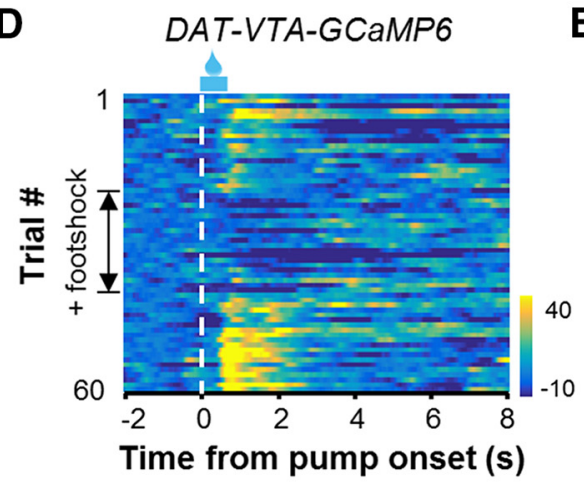

E

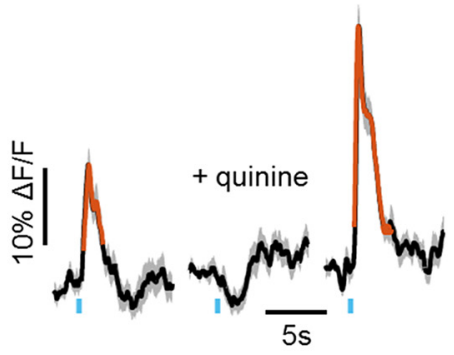

F

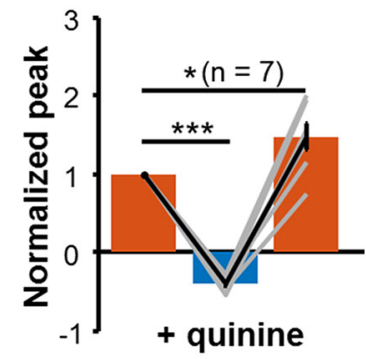

G

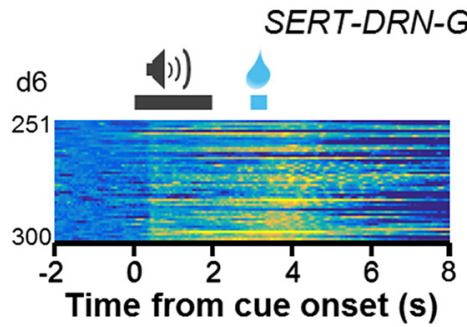

GCaMP6

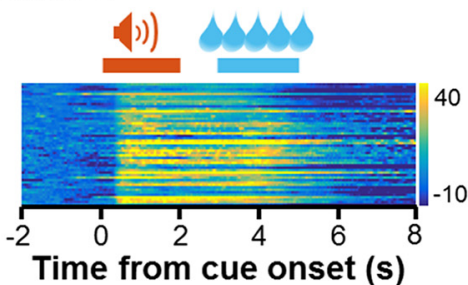

H SERT-DRN-GCaMPG

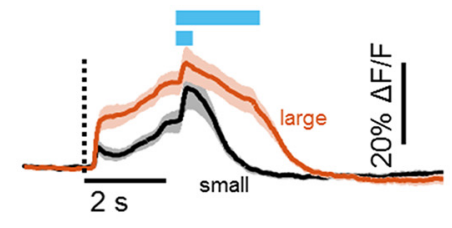

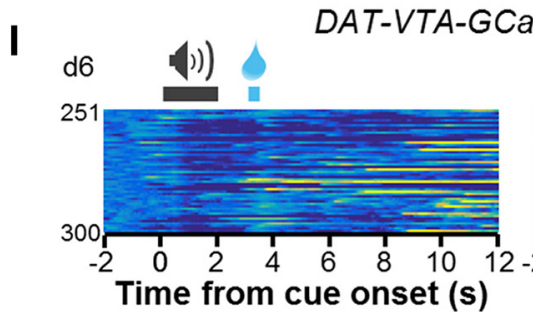

CaMP6

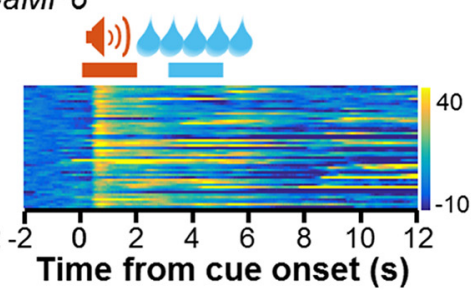

\section{J DAT-VTA-GCaMP6}

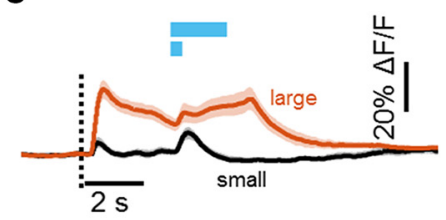

Figure 3. The effects of reward values on the responses of serotonin neurons and dopamine neurons. $A-C$, The effects of adding quinine on the sucrose-evoked $C^{2+}$ signal intensity of DRN serotonin neurons. The heatmap in $A$ illustrates the $\mathrm{Ca}^{2+}$ signals from a mouse across all trials. The sucrose concentration was held at $5 \%$ for 60 trials; quinine $(10 \mathrm{~mm})$ was added during trials $21-40$. The peri-event plots in $\boldsymbol{B}$ show the average $\mathrm{Ca}^{2+}$ signals to sucrose from the animal shown in $\boldsymbol{A}$ before, during, and after the quinine trials. Data are aligned to pump onset for liquid delivery. Population-level data in ( show the effect of mixing quinine with sucrose on the response of DRN serotonin neurons ( $n=6 \mathrm{SERT}$-DRN-GCaMP6 mice). Response amplitudes were normalized to those before quinine addition for each individual mouse. $\boldsymbol{D}-\boldsymbol{F}$, Heatmap representation $(\boldsymbol{D})$ and peri-event plot of $\mathrm{Ca}^{2+}$ signals $(\boldsymbol{E})$ from a representative mouse and population data $(\boldsymbol{F}, n=7$ DAT-VTA-GCaMP6 mice) showing the effects of adding quinine on the sucrose response of VTA dopamine neurons. $G$, Heatmaps illustrating the GCaMP changes $(\triangle F / F, \%)$ of DRN serotonin neurons to small (left) and large (right) rewards from a SERT-DRN-GCaMP6 mouse during the day 6 conditioning session. The trials of the two reward sizes were intermixed in pseudorandom order and sorted for illustration purposes. $\boldsymbol{H}$, Peri-event plots of the average $\mathrm{Ca}^{2+}$ signals of DRN serotonin neurons to the small (black) and large (red) rewards. $(n=8$ SERT-DRN-GCaMP6 mice). $\boldsymbol{I}, \boldsymbol{J}$, Heatmaps $(\boldsymbol{I})$ and average $\mathrm{Ca}^{2+}$ signals of VTA dopamine neurons $(\boldsymbol{J})$ during the day 6 conditioning session ( $n=7$ DAT-VTA-GCaMP6 mice). Error bars in $\left(\right.$ and $\boldsymbol{F}$ indicate SEM. ${ }^{*} p<0.05 ;{ }^{* *} p<0.01 ;{ }^{* * *} p<$ 0.001 ; n.s., not significant; multiple comparisons after repeated-measures one-way ANOVA. Shaded areas in $\boldsymbol{B}, \boldsymbol{E}, \boldsymbol{H}$, and $\boldsymbol{J}$ indicate $S E M$. Red and blue colors in $\boldsymbol{B}$ and $\boldsymbol{E}$ indicate significant increases and decreases from the baseline, respectively ( $p<0.05$, multivariate permutation tests). See Figures 3-1, 3-2, and 3-3 available at https://doi.org/10.1523/JNEUROSCI.1181-17.2017.f3-1, https://doi.org/10.1523/JNEUROSCI.1181-17.2017.f3-2, and https://doi.org/10.1523/JNEUROSCI.1181-17.2017.f3-3, respectively.

We examined how reward values affect the neuronal activity of serotonin neurons and dopamine neurons during Pavlovian conditioning. We coupled two auditory cues [a $4 \mathrm{kHz}$ pure tone (CS1), white noise (CS2)], respectively to a small reward and a large reward [0.5 s (US1) or $2.5 \mathrm{~s}$ (US2) of sucrose infusion]. Both SERT-DRN-GCaMP6 mice and DAT-VTA-GCaMP6 mice underwent six conditioning sessions, each of which consisted of 50 CS1-US1 pairs and 50 CS2-US2 pairs that were presented in a 
A

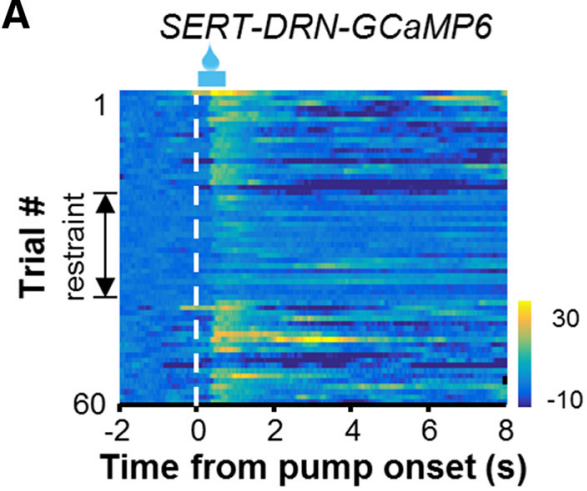

B

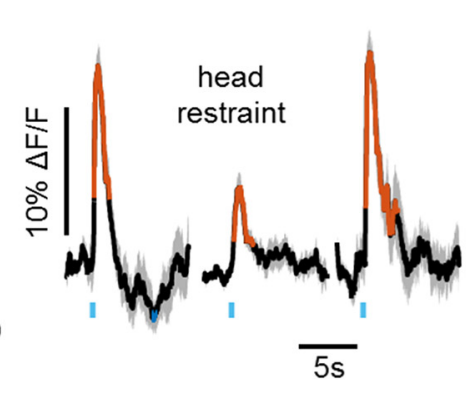

C

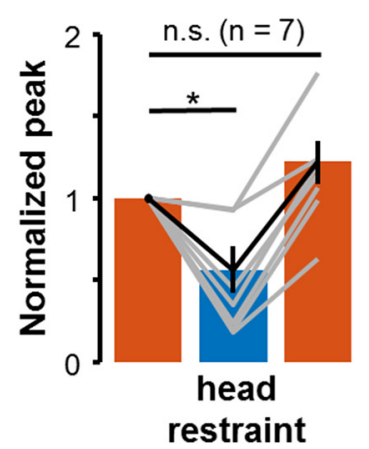

D

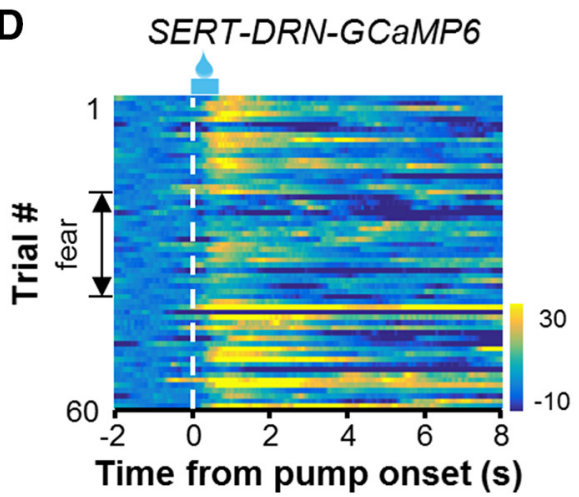

E

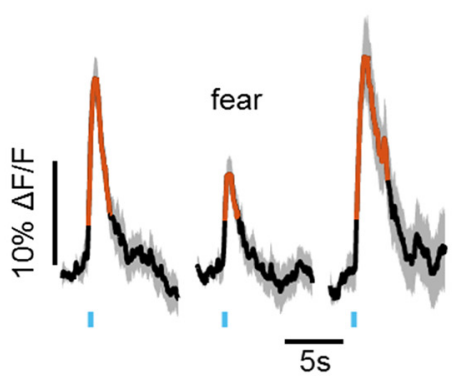

F

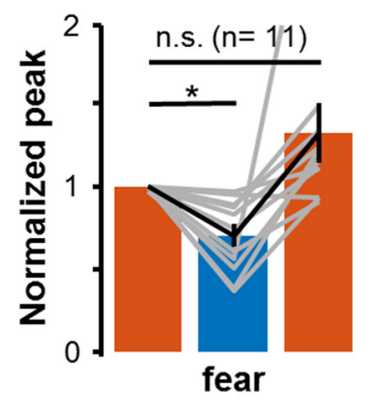

Figure 4. Acute stress decreases the reward response intensity of DRN serotonin neurons. $A$, A heatmap showing the effect of acutely restraining on the $C a^{2+}$ signal intensity of DRN serotonin neurons. The same amount of sucrose was delivered for 60 trials, and the mouse was head-restrained during trials $21-40 . B$, Peri-event plot of the average $C a^{2+}$ signals to sucrose from the animal shown in $\boldsymbol{A}$ before, during, and after the trials with head restraint. Data are aligned to pump onset for liquid delivery. $\boldsymbol{C}$, Population-level data showing the effect of head restraint on the response of DRN serotonin neurons ( $n=7$ SERT-DRN-GCaMP6 mice). Response amplitudes were normalized to those before head restraint for each individual mouse. $\boldsymbol{D}-\boldsymbol{F}$, The effects of a fearful context on the sucrose response of DRN serotonin neurons ( $n=11$ SERT-DRN-GCaMP6 mice). A total of 60 trials were recorded. After initial 20 trials of recording, a mouse was moved to a new chamber and given five random footshocks $\sim 10$ min before the recording of 20 trials recording sucrose responses. The mouse was then returned to the initial recording chamber and a final 20 trials were recorded. Error bars in $\boldsymbol{C}$ and $\boldsymbol{F}$ indicate SEM. ${ }^{*} p<0.05 ;{ }^{* *} p<0.01 ;$ n.S., not significant; multiple comparisons after repeated-measures one-way ANOVA. Shaded areas in $\boldsymbol{B}$ and $\boldsymbol{E}$ indicate SEM. Red and blue colors in $\boldsymbol{B}$ and $\boldsymbol{E}$ indicate significant increases and decreases from the baseline, respectively $(p<0.05$, multivariate permutation tests). See Figure 4-1 available at https://doi.org/10.1523/JNEUROSCI.1181-17.2017.f4-1.

pseudorandom order. For both serotonin neurons and dopamine neurons, the larger reward produced stronger $\mathrm{Ca}^{2+}$ signals and conditioned stronger activation by CS2 (Fig. 3G-J and Fig. 3-3A-H available at https://doi.org/10.1523/JNEUROSCI.1181-17.2017. f3-3). For serotonin neurons, the two cues predicting different reward sizes produced size-dependent responses after only two training sessions (Fig. $3 G, H$ and Fig. 3-3A-D, I,J available at https://doi.org/10.1523/JNEUROSCI.1181-17.2017.f3-3). For dopamine neurons, in contrast, the intensity of the activation only grew over time for the cue predicting the large reward; whereas the response strength of dopamine neurons to the cue predicting the small reward did not change (Fig. 3I, J, and Fig. 3-3E-H,K,L available at https://doi.org/10.1523/JNEUROSCI. 1181-17.2017.f3-3). Therefore, the particular reward sizes exert differential impacts on the serotonin neuron responses versus dopamine neuron responses. During the learning process, the response strengths of serotonin neurons more faithfully track reward values, whereas dopamine neurons prefer a larger reward.

\section{Acute stressors suppress the responses of serotonin neurons} and dopamine neurons to rewards and reward-predicting cues

We used two separate stressor treatments to study how acute stress affects the reward responses of DRN serotonin neurons and VTA dopamine neurons in mice that had not undergone Pavlov- ian conditioning. We challenged mice with a head restraint, which, unlike the quinine stressor, was not perfectly aligned with reward delivery in time. In a single stress session, we recorded sucrose-evoked $\mathrm{Ca}^{2+}$ signals for 20 trials in freely behaving mice, in 20 additional trials during which mice were head-restrained, and finally in 20 trials in freely behaving mice that had been released from the head restraint (Fig. $4 A$ ). For serotonin neurons, head restraint reduced the average intensity of the sucrose response by nearly $50 \%\left(n=7\right.$ mice; $F_{(2,18)}=11.14, p=0.0012$, repeated-measures one-way ANOVA with "stress" as the withinsubject factor), and this decrease was fully reversible upon release (Fig. 4C).

A stress experience that occurs some period before reward delivery might also have an effect on the reward responses of neurons. To explore this idea, we devised experiments in which we observed differences in the $\mathrm{Ca}^{2+}$ signals between normal and fearful context. We first recorded sucrose-evoked responses of serotonin neurons for 20 trials in a "normal" recording chamber. To create a fearful context, we placed the mouse in a new chamber, randomly delivered five footshocks ( $1 \mathrm{~s}, 0.7 \mathrm{~mA})$, and after 10 min recorded sucrose responses for 20 trials. Finally, we returned the mouse back to the initial normal chamber and recorded responses for 20 normal sucrose delivery trials. The intensity of the sucrose-evoked $\mathrm{Ca}^{2+}$ signals was reversibly reduced by $\sim 30 \%$ in the chamber associated with prior footshocks $(n=11$ mice; Fig. 
A

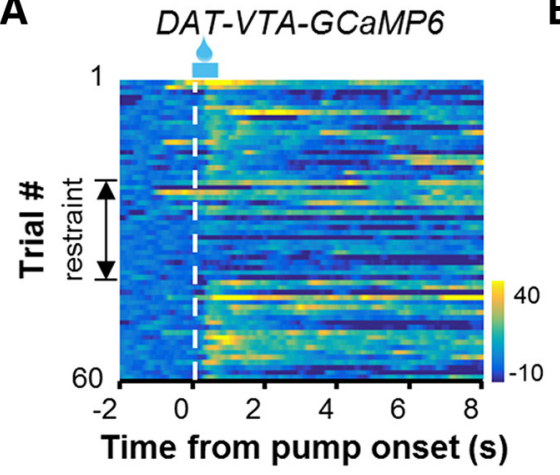

B

E

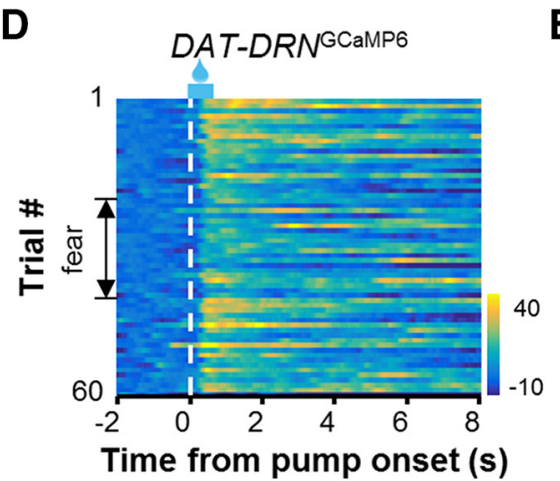

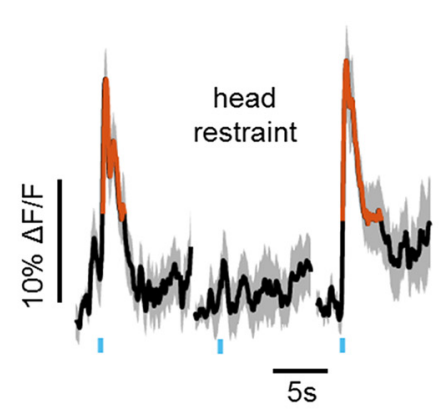

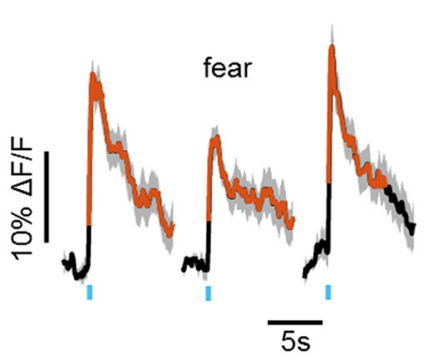

C

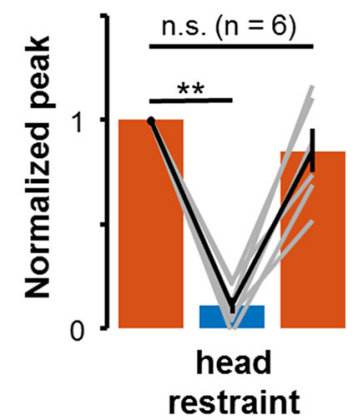

F

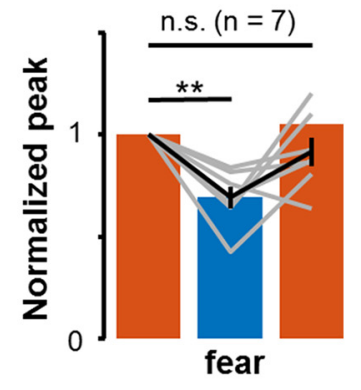

Figure 5. Acute stress decreases the reward response intensity of VTA dopamine neurons. $\boldsymbol{A}-\boldsymbol{C}$, The effect of acute head-restraint on the sucrose response of VTA dopamine neurons ( $n=6$ mice). $\boldsymbol{D}-\boldsymbol{F}$, The effect of placing an animal in a fearful context on the sucrose response of VTA dopamine neurons $\left(n=7\right.$ mice). Error bars in $\boldsymbol{C}$ and $\boldsymbol{F}$ indicate $\mathbf{S E M}$. ${ }^{*} p<0.05$; ${ }^{* *} p<0.01 ; n .5 .$, not significant; multiple comparisons after repeated-measures one-way ANOVA. Shaded areas in $\boldsymbol{B}$ and $\boldsymbol{E}$ indicate $S E M$. Red and blue colors in $\boldsymbol{B}$ and $\boldsymbol{E}$ indicate significant increases and decreases from the baseline, respectively ( $p<0.05$, multivariate permutation tests).

$4 D-F ; F_{(2,30)}=14.36, p=0.0002$, repeated-measures one-way ANOVA with stress as the within-subject factor). Thus, prior stress can strongly decrease the reward responses of DRN serotonin neurons. Consistent with the reduction in $\mathrm{Ca}^{2+}$ signals, introducing mice into the fearful context disrupted the locomotor response pattern associated with sucrose delivery (Fig. 4-1 available at https://doi.org/10.1523/JNEUROSCI.1181-17.2017.f4-1).

Stressors also suppressed the sucrose-evoked $\mathrm{Ca}^{2+}$ signals of VTA dopamine neurons. Head restraint reduced the sucroseevoked responses of dopamine neurons by $>90 \%$ ( $n=6$ mice; Fig. $5 A-C ; F_{(2,15)}=9.333, p=0.0055$, repeated-measures oneway ANOVA with stress as the within-subject factor). For the chamber associated with prior footshock, the intensity of the responses of dopamine neurons was reduced by approximately one-third $\left(n=7\right.$ mice; Fig. $5 D-F ; F_{(2,18)}=8.857, p=0.0084$, repeatedmeasures one-way ANOVA with stress as the within-subject factor), suggesting that a fearful context can also decrease the reward responses of VTA dopamine neurons.

We further investigated how stress associated with head restraint and a fearful context would affect neuronal responses to reward-predicting cues. Mice completed multiple sessions (100 trials per session) of Pavlovian conditioning to establish strong associations between a cue and a large reward (2.5 s sucrose delivery). For both DRN serotonin neurons and VTA dopamine neurons, acute head restraint and fearful context reversibly reduced the intensity of responses to the cue by $\sim 70 \%$ and $50 \%$, respectively $\left(n=6\right.$ mice; Fig. $6 A-C ; F_{(2,15)}=11.14, p=0.012$; $n=6$ mice; Fig. $6 D-F ; F_{(2,15)}=8.333, p=0.012 ; n=6$ mice; Fig. $7 A-C ; F_{(2,15)}=9.333, p=0.0055 ; n=8$ mice; Fig. $7 D-F ; F_{(2,21)}=$ 9.75, $p=0.0048$; repeated-measures one-way ANOVA with stress as the within-subject factor). Consistent with the reduction in $\mathrm{Ca}^{2+}$ signals, introducing mice into the fearful context disrupted the locomotor response pattern associated with the cuesucrose stimulus pairs (Fig. 6-1 available at https://doi.org/10. 1523/JNEUROSCI.1181-17.2017.f6-1). Therefore, stress reduces the intensity of the responses of both DRN serotonin neurons and VTA dopamine neurons and affects the pattern of conditioned responses to reward-predicting cues at the behavioral level.

\section{Discussion}

Using fiber photometry of $\mathrm{Ca}^{2+}$ signals, we examined how learning and acute stress affect the responses of DRN 5-HT neurons and VTA dopamine neurons of mice to both reward and rewardpredicting cues over a long experimental period. We first compared how DRN serotonin neurons and VTA dopamine neurons respond differently during the entire process of appetitive Pavlovian conditioning. Important reward parameters such as reward value and availability determined the response intensity and the response pattern for both of the neuron populations. By applying various aversive stimuli, we further revealed that stress suppresses the response intensity of both serotonin neurons and dopamine neurons to rewards and to reward-predicting cues. Our findings indicate that reward responses adapt dynamically during reward associative learning and stressful contexts, suggesting a role of serotonin neurons and dopamine neurons in mediating the effects of stress on reward-related behaviors.

Learning gradually shapes the reward responses of serotonin neurons and dopamine neurons into different patterns By tracking the neuronal activity throughout the entire process of Pavlovian conditioning (up to 600 trials over $6 \mathrm{~d}$ ), we were able to observe several aspects of how learning shapes the response pat- 
A

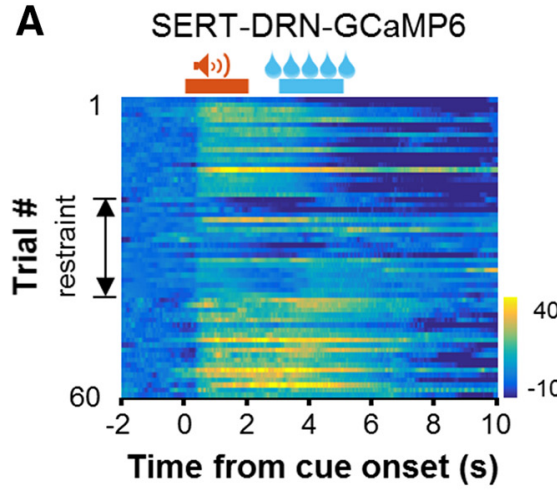

D SERT-DRN-GCaMP6

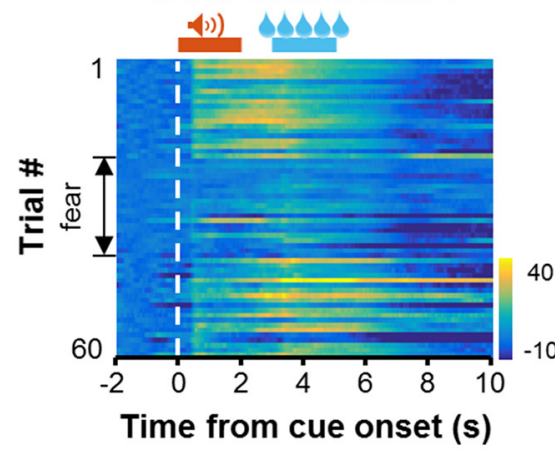

B

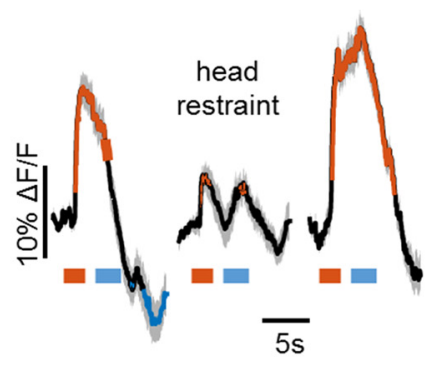

E

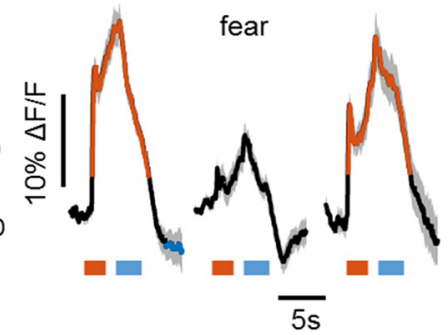

C

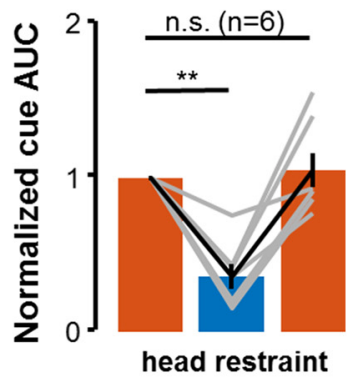

$\mathbf{F}$

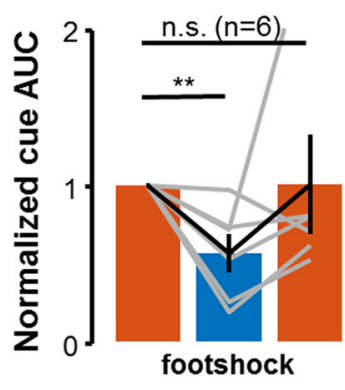

Figure 6. Acute stress decreases the conditioned responses of DRN serotonin to reward-predicting cues. $\boldsymbol{A}-\boldsymbol{C}$, The effect of head restraint on the conditioned responses of DRN serotonin neurons to a cue that was associated with sucrose delivery. $\boldsymbol{A}$, Heatmap representation of $\mathrm{Ca}^{2+}$ signals; $(\boldsymbol{B})$ average plot of $\mathrm{Ca}^{2+}$ signals before, during, and after the trials with head restraint; $(\boldsymbol{C})$ population data $\left(n=7\right.$ SERT-DRN-GCaMP6 mice). $\boldsymbol{D}-\boldsymbol{F}$, Heatmap $(\boldsymbol{D})$ and average $\mathrm{Ca}^{2+}$ signals $(\boldsymbol{E})$ of a mouse and population data $(\boldsymbol{F})$ showing the effect of a fearful context on the responses of DRN serotonin neurons to reward-predicting cues $\left(n=6\right.$ mice). Error bars in $\boldsymbol{C}$ and $\boldsymbol{F}$ indicate SEM. ${ }^{* *} p<0.01$; n.S., not significant; multiple comparisons after repeated-measures one-way ANOVA. Shaded areas in $\boldsymbol{B}$ and $\boldsymbol{E}$ indicate SEM. Red and blue colors in $\boldsymbol{B}$ and $\boldsymbol{E}$ indicate significant increases and decreases from the baseline, respectively $(p<0.05$, multivariate permutation tests). See Figure $6-1$ available at https://doi.org/10.1523/JNEUROSCI.1181-17.2017.f6-1.

terns of DRN serotonin neurons and VTA dopamine neurons. Initially, both types of neurons respond to primary rewards. After $\sim 170$ trials of Pavlovian conditioning, the response pattern of serotonin neurons became clearly different from that of dopamine neurons. Most prominently, for DRN serotonin neurons, the process of Pavlovian conditioning gradually leads to increasingly stronger ramp-up of $\mathrm{Ca}^{2+}$ signals following the onset of the reward-predicting cue (CS); the peak intensity of activation eventually coincided with the delivery of the primary reward (US). In contrast, for dopamine neurons, Pavlovian conditioning leads to $\mathrm{a} \mathrm{Ca}^{2+}$ response to the CS, but a substantially weaker $\mathrm{Ca}^{2+}$ response to the US (Fig. 1). Moreover, reward availability has different effects on serotonin neurons and dopamine neurons. Reward omission reduces the responses of both serotonin neurons and dopamine neurons. For serotonin neurons, reward reinstatement leads to a gradual recovery in the previously established ramp-up response pattern. For dopamine neurons, reward reinstatement produces a dramatically stronger activation when an omitted reward is redelivered (Fig. 2). Testing with two reward sizes suggests that serotonin neurons more faithfully represent the "absolute" value of a reward, whereas dopamine neurons represent the "relative" value of the reward (Fig. 3).

Our observation of distinct response patterns suggests that serotonin neurons and dopamine neurons perform different functions in reward processing. Our observations support the theory that transient dopamine levels encode information about the difference between an actual reward and an expected reward. As proposed by the temporal difference-lambda learning model, the dopamine signals may serve as a "credit" that can be assigned to distant states and actions in a manner that is inversely proportional to time difference (Schultz et al., 1997; Montague et al., 2004; Cohen et al., 2012; Eshel et al., 2015; Sadacca et al., 2016). This reward credit may in turn drive the changes in associative strength between neurons encoding the CS (cue) and those encoding the US (primary reward; Rescorla and Wagner, 1972; Sutton, 1988; Schultz et al., 1997; Montague et al., 2004). However, the information carried by dopamine neurons after learning is limited in two major ways. First, they cannot respond positively to the acquisition of an expected reward, although receiving such a reward remains pleasurable for an animal. Second, dopamine neurons respond transiently to a reward-predicting cue, so their activity cannot continuously guide reward-related behavior during the delay between the CS and US. After learning, unlike dopamine neurons, serotonin neurons continue to respond to the primary reward. This suggests that serotonin neurons contribute to the accurate evaluation of the acquisition status of a reward. Moreover, the ramp-up activation of serotonin neurons during the reward anticipatory phase might be important for promoting patience during a "waiting to obtain a reward" period (Miyazaki et al., 2011, 2014; Fonseca et al., 2015). However, during Pavlovian conditioning, larger reward sizes produce stronger $\mathrm{Ca}^{2+}$ signals during the waiting period, even though the duration of waiting period remains the same. This suggests that the activity of serotonin neurons encodes information beyond waiting duration, possibly including information related to attention allocation or related to the confidence level that an organism ascribes to 
A

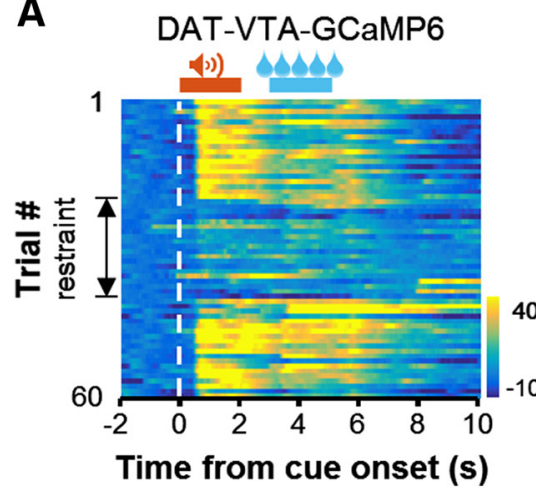

D

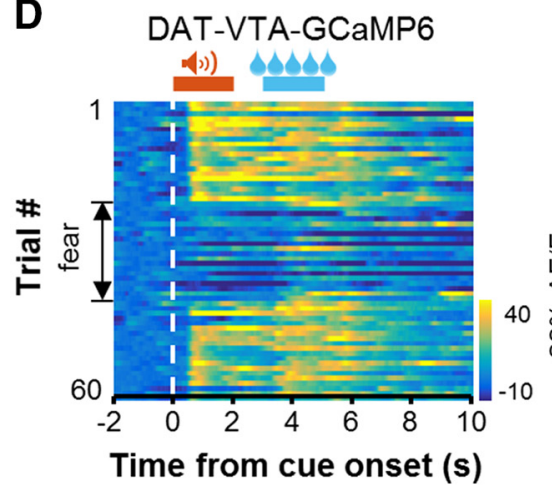

B

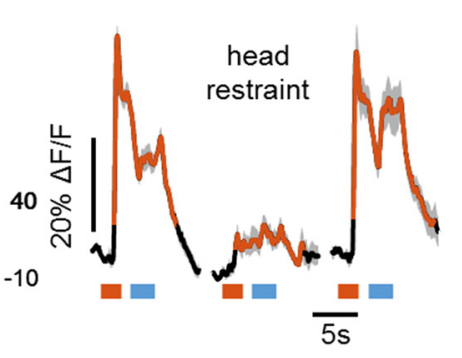

$\mathbf{E}$

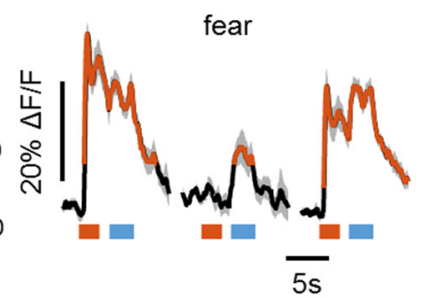

C

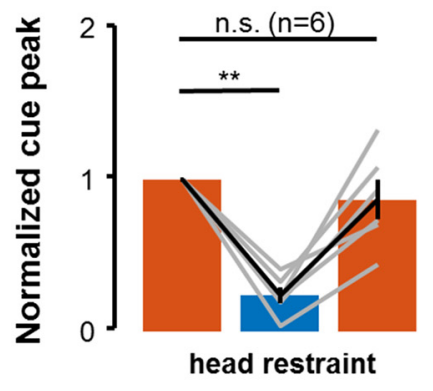

$\mathbf{F}$

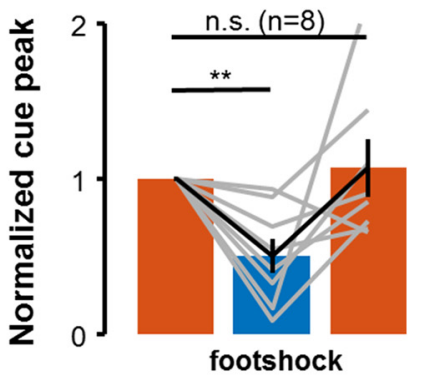

Figure 7. Acute stress decreases the conditioned responses of VTA dopamine neurons to reward-predicting cues. $\boldsymbol{A}-\boldsymbol{C}$, Heatmap $(\boldsymbol{A})$ and peri-event plot of $\mathrm{Ca}{ }^{2+}$ signals $(\boldsymbol{B})$ of a representative mouse and population data $(\boldsymbol{C})$ showing the effect of head restraint on the responses of VTA dopamine neurons to reward-associated cues $(n=6$ DAT-VTA-GCaMP6 mice). D-F, Heatmap (D) and peri-event plot of $\mathrm{Ca}^{2+}$ signals $(\boldsymbol{E})$ of a mouse and population data $(\boldsymbol{F})$ showing the effect of fearful context on the responses of VTA dopamine neurons to reward-predicting cues $(n=8$ mice). Error bars in $\boldsymbol{E}$ and $\boldsymbol{F}$ indicate SEM. ${ }^{* *} p<0.01$; n.S., not significant; multiple comparisons after repeated-measures one-way ANOVA. Shaded areas in $\boldsymbol{B}$ and $\boldsymbol{E}$ indicate SEM. Red and blue colors in $\boldsymbol{B}$ and $\boldsymbol{E}$ indicate significant increases and decreases from the baseline, respectively ( $p<0.05$, multivariate permutation tests).

the probability of receiving a reward in a given state (Pearce and Hall, 1980; Cardinal et al., 2002; Pearce and Mackintosh, 2010; Luo et al., 2016; Matias et al., 2017).

The response patterns of DRN serotonin neurons revealed in this study differ substantially from those reported in a recent study (Matias et al., 2017). First, the Matias et al. (2017) study showed that after conditioning DRN serotonin neurons are transiently activated by reward-predicting cues and lack responses to a fully predicted reward, whereas we found that these neurons exhibit a ramp-up activation pattern during the reward anticipatory phase and remain responsive to the primary reward. Second, they showed that the serotonin neurons were activated when a fully predicted reward was omitted, whereas we found that reward omission gradually reduced the response of these neurons to the reward-predicting cue and rapidly abolished the response during the period of reward omission. After learning, the ramp-up pattern of serotonin neurons shown in our study resembles the pattern of tonic spike firing by putative serotonin neurons in the DRN noted in several previous reports (Nakamura et al., 2008; Bromberg-Martin et al., 2010; Miyazaki et al., 2011; Liu et al., 2014; Li et al., 2016). It should be noted that single-unit recordings showed that a small number of putative serotonergic neurons in the DRN prefer small rewards or are inhibited by rewards (Nakamura et al., 2008; Li et al., 2016). Fiber photometry only detects the activity of populations of cells and may thus be inadequately sensitive to detect minor response patterns. The $\mathrm{Ca}^{2+}$ signal patterns of VTA dopamine neurons revealed by our fiber photometry technique are highly similar to the spike firing patterns and $\mathrm{Ca}^{2+}$ signal patterns reported for the VTA dopamine neurons in primates and rodents (Schultz et al., 1997; Montague et al., 2004; Nakamura et al., 2008; Cohen et al., 2012; Eshel et al., 2015; Menegas et al., 2017). These similarities support the validity of our fiber photometry approach. Therefore, our findings do not fully agree with the suggestion that DRN serotonin neurons encode "unsigned" (both positive and negative) reward prediction error (Matias et al., 2017). We note that Matias et al. (2017) recorded from chronically head-fixed mice, whereas we recorded from freely behaving mice. It will be interesting in future studies to investigate whether the difference between these two recording conditions results in different response patterns.

\section{Stressors reduce the reward responses of DRN serotonin neurons and VTA dopamine neurons}

A key finding of the present study is that various stressors can substantially suppress the response intensities of both DRN serotonin neurons and VTA dopamine neurons to a primary reward and to reward-predicting cues. Mixing the sucrose reward with quinine completely abolished the sucrose-evoked $\mathrm{Ca}^{2+}$ signals of both serotonin neurons and dopamine neurons. For both types of neurons, head restraint or the fear context associated with prior footshocks substantially reduced sucrose-associated $\mathrm{Ca}^{2+}$ signals. After an animal learned to associate a cue with a reward, head restraint and fearful context also significantly reduced the excitatory responses to the reward-predicting cue. These stressors involve different sensory modalities, suggesting that stress generally suppresses the responses of serotonin neurons and dopamine neurons to reward and to reward-predicting cues.

An important implication from our study may be the indication that serotonin neurons and dopamine neurons function to integrate information about reward value and cost. Our data do 
not support the notion that 5-HT neurons at the population level positively encode aversiveness. It has been reported that airpuffs briefly activate many 5-HT neurons in the DRN of head-fixed mice (Cohen et al., 2015). It remains unclear whether the airpuffevoked responses are associated with the aversive nature of airpuff or with its strong sensory saliency (Horvitz et al., 1997; Horvitz, 2000). Our previous study showed that various rewarding stimuli activate DRN serotonin neurons, whereas quinine and footshock induce mild reductions in the intensity of population-level $\mathrm{Ca}^{2+}$ signals (Li et al., 2016). Here, we further showed that coupling a cue to quinine resulted in reduction of $\mathrm{Ca}^{2+}$ signals from the baseline. More importantly, we demonstrated that different stressors inhibited the reward-associated $\mathrm{Ca}^{2+}$ signals of serotonin neurons. These findings support our recent argument that the activity levels of serotonin neurons effectively correspond to "beneficialness", how beneficial the current environmental state represents to an animal (Luo et al., 2016). The beneficialness is determined by "net benefit", which can be calculated as the reward values minus costs. The circuit underlying this calculation remains to be dissected, although the lateral habenula $(\mathrm{LHb})$ represents an obvious source for the stress (i.e., cost) signals. LHb neurons are activated by stressors and, via GABAergic relays, inhibit DRN serotonin neurons and VTA dopamine neurons (Ji and Shepard, 2007; Li et al., 2013; Proulx et al., 2014; Sego et al., 2014; Hu, 2016; Wang et al., 2017; Zhou et al., 2017).

Our study revealed that learning and reward parameters exert different effects on the responses of DRN serotonin neurons and VTA dopamine neurons and suggests that these two neuron populations perform complementary roles in reward processing. Moreover, we demonstrated that stress substantially reduces the reward responses of DRN serotonin neurons and VTA dopamine neurons. Anhedonia is a key symptom of depression, and stress represents one of the most reliable predictors of depression in humans (Kendler et al., 1999; Gold and Chrousos, 2002; Hammen, 2005). The negative effect of stress on reward responses provides evidence to support the association of acute stress and anhedonia (Bogdan and Pizzagalli, 2006; Chrousos, 2009; Hollon et al., 2015), suggesting that some reduction in the reward responses of both serotonin neurons and dopamine neurons might underlie stress-induced anhedonia. Enhancing the reward sensitivity of these two important neuron populations might ameliorate psychiatric disorders associated with stress-induced anhedonia.

\section{References}

Amat J, Baratta MV, Paul E, Bland ST, Watkins LR, Maier SF (2005) Medial prefrontal cortex determines how stressor controllability affects behavior and dorsal raphe nucleus. Nat Neurosci 8:365-371. CrossRef Medline

Anisman H, Zacharko RM (1990) Multiple neurochemical and behavioral consequences of stressors: implications for depression. Pharmacol Ther 46:119-136. CrossRef Medline

Bekris S, Antoniou K, Daskas S, Papadopoulou-Daifoti Z (2005) Behavioural and neurochemical effects induced by chronic mild stress applied to two different rat strains. Behav Brain Res 161:45-59. CrossRef Medline

Berghorst LH, Bogdan R, Frank MJ, Pizzagalli DA (2013) Acute stress selectively reduces reward sensitivity. Front Hum Neurosci 7:133. CrossRef Medline

Bogdan R, Pizzagalli DA (2006) Acute stress reduces reward responsiveness: implications for depression. Biol Psychiatry 60:1147-1154. CrossRef Medline

Born JM, Lemmens SG, Rutters F, Nieuwenhuizen AG, Formisano E, Goebel R, Westerterp-Plantenga MS (2010) Acute stress and food-related reward activation in the brain during food choice during eating in the absence of hunger. Int J Obes (Lond) 34:172-181. CrossRef Medline

Bromberg-Martin ES, Hikosaka O, Nakamura K (2010) Coding of task reward value in the dorsal raphe nucleus. J Neurosci 30:6262-6272. CrossRef Medline

Cardinal RN, Parkinson JA, Hall J, Everitt BJ (2002) Emotion and motivation: the role of the amygdala, ventral striatum, and prefrontal cortex. Neurosci Biobehav Rev 26:321-352. CrossRef Medline

Chen TW, Wardill TJ, Sun Y, Pulver SR, Renninger SL, Baohan A, Schreiter ER, Kerr RA, Orger MB, Jayaraman V, Looger LL, Svoboda K, Kim DS (2013) Ultrasensitive fluorescent proteins for imaging neuronal activity. Nature 499:295-300. CrossRef Medline

Chrousos GP (2009) Stress and disorders of the stress system. Nat Rev Endocrinol 5:374-381. CrossRef Medline

Cohen JY, Haesler S, Vong L, Lowell BB, Uchida N (2012) Neuron-typespecific signals for reward and punishment in the ventral tegmental area. Nature 482:85-88. CrossRef Medline

Cohen JY, Amoroso MW, Uchida N (2015) Serotonergic neurons signal reward and punishment on multiple timescales. eLife 4:e06346. CrossRef Medline

Correia PA, Lottem E, Banerjee D, Machado AS, Carey MR, Mainen ZF (2017) Transient inhibition and long-term facilitation of locomotion by phasic optogenetic activation of serotonin neurons. eLife 6:e20975. CrossRef Medline

de Kloet ER, Joëls M, Holsboer F (2005) Stress and the brain: from adaptation to disease. Nat Rev Neurosci 6:463-475. CrossRef Medline

Eshel N, Bukwich M, Rao V, Hemmelder V, Tian J, Uchida N (2015) Arithmetic and local circuitry underlying dopamine prediction errors. Nature 525:243-246. CrossRef Medline

Fonseca MS, Murakami M, Mainen ZF (2015) Activation of dorsal raphe serotonergic neurons promotes waiting but is not reinforcing. Curr Biol 25:306-315. CrossRef Medline

Gold PW, Chrousos GP (2002) Organization of the stress system and its dysregulation in melancholic and atypical depression: high vs low CRH/NE states. Mol Psychiatry 7:254-275. CrossRef Medline

Groppe DM, Urbach TP, Kutas M (2011) Mass univariate analysis of eventrelated brain potentials/fields II: simulation studies. Psychophysiology 48:1726-1737. CrossRef Medline

Gunaydin LA, Grosenick L, Finkelstein JC, Kauvar IV, Fenno LE, Adhikari A, Lammel S, Mirzabekov JJ, Airan RD, Zalocusky KA, Tye KM, Anikeeva P, Malenka RC, Deisseroth K (2014) Natural neural projection dynamics underlying social behavior. Cell 157:1535-1551. CrossRef Medline

Guo Q, Zhou J, Feng Q, Lin R, Gong H, Luo Q, Zeng S, Luo M, Fu L (2015) Multi-channel fiber photometry for population neuronal activity recording. Biomed Opt Express 6:3919-3931. CrossRef Medline

Hammen C (2005) Stress and depression. Annu Rev Clin Psychol 1:293319. CrossRef Medline

Hayashi K, Nakao K, Nakamura K (2015) Appetitive and aversive information coding in the primate dorsal raphe nucleus. J Neurosci 35:61956208. CrossRef Medline

Hintiryan H, Hayes UL, Chambers KC (2006) Intraoral cheek fistulae: a refined technique. Lab Anim 40:456-464. CrossRef Medline

Hollerman JR, Schultz W (1998) Dopamine neurons report an error in the temporal prediction of reward during learning. Nat Neurosci 1:304-309. CrossRef Medline

Hollon NG, Burgeno LM, Phillips PE (2015) Stress effects on the neural substrates of motivated behavior. Nat Neurosci 18:1405-1412. CrossRef Medline

Horvitz JC (2000) Mesolimbocortical and nigrostriatal dopamine responses to salient non-reward events. Neuroscience 96:651-656. CrossRef Medline

Horvitz JC, Stewart T, Jacobs BL (1997) Burst activity of ventral tegmental dopamine neurons is elicited by sensory stimuli in the awake cat. Brain Res 759:251-258. CrossRef Medline

Hu H (2016) Reward and aversion. Annu Rev Neurosci 39:297-324. CrossRef Medline

Ji H, Shepard PD (2007) Lateral habenula stimulation inhibits rat midbrain dopamine neurons through a $\mathrm{GABA}_{\mathrm{A}}$ receptor-mediated mechanism. J Neurosci 27:6923-6930. CrossRef Medline

Kendler KS, Karkowski LM, Prescott CA (1999) Causal relationship between stressful life events and the onset of major depression. Am J Psychiatry 156:837-841. CrossRef Medline

Kumar P, Berghorst LH, Nickerson LD, Dutra SJ, Goer FK, Greve DN, Pizzagalli DA (2014) Differential effects of acute stress on anticipatory and consummatory phases of reward processing. Neuroscience 266:112. CrossRef Medline 
Li K, Zhou T, Liao L, Yang Z, Wong C, Henn F, Malinow R, Yates JR 3rd, Hu H (2013) $\beta$ CaMKII in lateral habenula mediates core symptoms of depression. Science 341:1016-1020. CrossRef Medline

Li Y, Zhong W, Wang D, Feng Q, Liu Z, Zhou J, Jia C, Hu F, Zeng J, Guo Q, Fu L, Luo M (2016) Serotonin neurons in the dorsal raphe nucleus encode reward signals. Nat Commun 7:10503. CrossRef Medline

Liu Z, Zhou J, Li Y, Hu F, Lu Y, Ma M, Feng Q, Zhang JE, Wang D, Zeng J, Bao J, Kim JY, Chen ZF, El Mestikawy S, Luo M (2014) Dorsal raphe neurons signal reward through 5-HT and glutamate. Neuron 81:1360-1374. CrossRef Medline

Luo M, Li Y, Zhong W (2016) Do dorsal raphe 5-HT neurons encode "beneficialness"? Neurobiol Learn Mem 135:40-49. CrossRef Medline

Matias S, Lottem E, Dugué GP, Mainen ZF (2017) Activity patterns of serotonin neurons underlying cognitive flexibility. eLife 6:e20552. CrossRef Medline

Matsumoto M, Hikosaka O (2009) Two types of dopamine neuron distinctly convey positive and negative motivational signals. Nature 459: 837-841. CrossRef Medline

Menegas W, Babayan BM, Uchida N, Watabe-Uchida M (2017) Opposite initialization to novel cues in dopamine signaling in ventral and posterior striatum in mice. eLife 6:e21886. CrossRef Medline

Miyazaki K, Miyazaki KW, Doya K (2011) Activation of dorsal raphe serotonin neurons underlies waiting for delayed rewards. J Neurosci 31:469479. CrossRef Medline

Miyazaki KW, Miyazaki K, Tanaka KF, Yamanaka A, Takahashi A, Tabuchi S, Doya K (2014) Optogenetic activation of dorsal raphe serotonin neurons enhances patience for future rewards. Curr Biol 24:2033-2040. CrossRef Medline

Montague PR, Hyman SE, Cohen JD (2004) Computational roles for dopamine in behavioural control. Nature 431:760-767. CrossRef Medline

Nakamura K (2013) The role of the dorsal raphe nucleus in reward-seeking behavior. Front Integr Neurosci 7:60. CrossRef Medline

Nakamura K, Matsumoto M, Hikosaka O (2008) Reward-dependent modulation of neuronal activity in the primate dorsal raphe nucleus. J Neurosci 28:5331-5343. CrossRef Medline

Pearce JM, Hall G (1980) A model for Pavlovian learning: variations in the effectiveness of conditioned but not of unconditioned stimuli. Psychological review 87:532-552. CrossRef Medline

Pearce JM, Mackintosh NJ (2010) Two theories of attention: a review and a possible integration. In: Attention and associative learning: from brain to behaviour (Mitchell CJ, Le Pelley ME, eds), pp 11-39. Oxford, UK: Oxford UP.

Pollak Dorocic I, Fürth D, Xuan Y, Johansson Y, Pozzi L, Silberberg G, Carlén
M, Meletis K (2014) A whole-brain atlas of inputs to serotonergic neurons of the dorsal and median raphe nuclei. Neuron 83:663-678. CrossRef Medline

Porcelli AJ, Lewis AH, Delgado MR (2012) Acute stress influences neural circuits of reward processing. Front Neurosci 6:157. CrossRef Medline

Proulx CD, Hikosaka O, Malinow R (2014) Reward processing by the lateral habenula in normal and depressive behaviors. Nat Neurosci 17:11461152. CrossRef Medline

Rescorla RA, Wagner AW (1972) A theory of Pavlovian conditioning: variations in the effectiveness of reinforcement and nonreinforcement. In: Classical conditioning II: current research and theory (Black AH, Prokasy WF, eds), pp 64-99. New York: Appleton-Century-Crofts.

Sadacca BF, Jones JL, Schoenbaum G (2016) Midbrain dopamine neurons compute inferred and cached value prediction errors in a common framework. eLife 5:e13665. CrossRef Medline

Schneiderman N, Ironson G, Siegel SD (2005) Stress and health: psychological, behavioral, and biological determinants. Annu Rev Clin Psychol 1:607-628. CrossRef Medline

Schultz W, Dayan P, Montague PR (1997) A neural substrate of prediction and reward. Science 275:1593-1599. CrossRef Medline

Sego C, Gonçalves L, Lima L, Furigo IC, Donato J Jr, Metzger M (2014) Lateral habenula and the rostromedial tegmental nucleus innervate neurochemically distinct subdivisions of the dorsal raphe nucleus in the rat. J Comp Neurol 522:1454-1484. CrossRef Medline

Sutton RS (1988) Learning to predict by the methods of temporal differences. Mach Learn 3:9-44. CrossRef

Wang D, Li Y, Feng Q, Guo Q, Zhou J, Luo M (2017) Learning shapes the aversion and reward responses of lateral habenula neurons. eLife 6:e23045. CrossRef Medline

Watabe-Uchida M, Zhu L, Ogawa SK, Vamanrao A, Uchida N (2012) Whole-brain mapping of direct inputs to midbrain dopamine neurons. Neuron 74:858-873. CrossRef Medline

Weissbourd B, Ren J, DeLoach KE, Guenthner CJ, Miyamichi K, Luo L (2014) Presynaptic partners of dorsal raphe serotonergic and GABAergic neurons. Neuron 83:645-662. CrossRef Medline

Zhang X, Beaulieu JM, Sotnikova TD, Gainetdinov RR, Caron MG (2004) Tryptophan hydroxylase-2 controls brain serotonin synthesis. Science 305:217. CrossRef Medline

Zhou L, Liu MZ, Li Q, Deng J, Mu D, Sun YG (2017) Organization of functional long-range circuits controlling the activity of serotonergic neurons in the dorsal raphe nucleus. Cell Rep 18:3018-3032. CrossRef Medline 